NISSUNA UMANA INVESTIGAZIONE SI PUO DIMANDARE VERA SCIENZIA S'ESSA NON PASSA PER LE MATEMATICHE DIMOSTRAZIONI LEONARDO DA VINCI

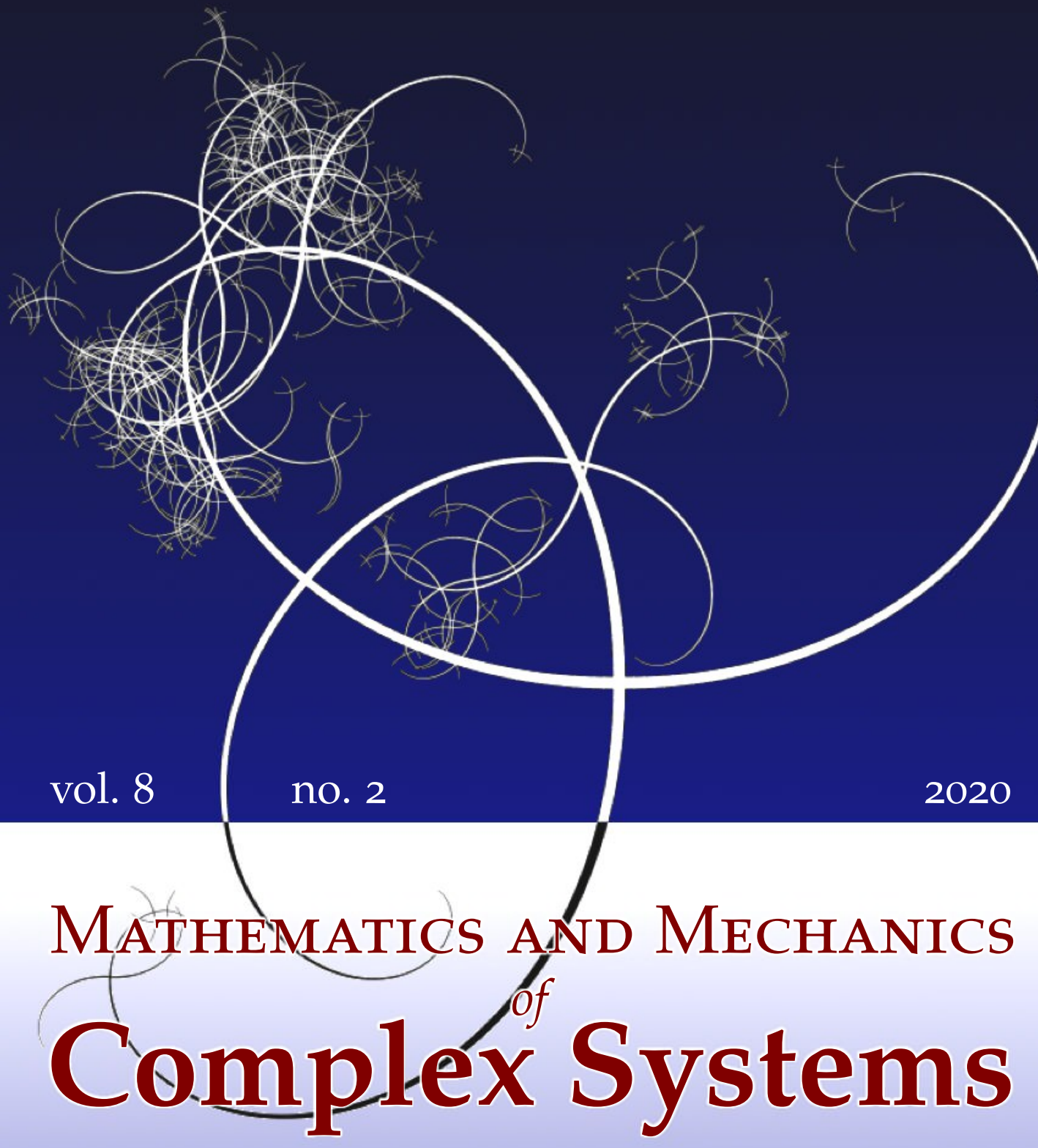

Angelo Luongo And Francesco D' Annibale

MODELING THE LINEAR DYNAMICS

OF CONTINUOUS VISCOELASTIC SYSTEMS

ON THEIR INFINITE-DIMENSIONAL CENTRAL SUBSPACE 


\title{
MODELING THE LINEAR DYNAMICS OF CONTINUOUS VISCOELASTIC SYSTEMS ON THEIR INFINITE-DIMENSIONAL CENTRAL SUBSPACE
}

\author{
Angelo Luongo And Francesco D’Annibale
}

\begin{abstract}
A metamodel of linear viscoelastic continuum is formulated. Internal variables, of arbitrary number, are introduced to describe the viscous part of the strain, and a wide class of constitutive laws, suggested by rheological models, is considered. The spectral properties of the system are discussed. Based on the separation of the eigenvalues occurring when the viscous moduli are small, the system is reduced to its infinite-dimensional central subspace, on which the steady dynamics takes place. Both the center manifold method and the multiple scales method are used to build the reduced model, which is formulated in terms of the only observable variables. Examples relevant to one-, two-, and three-dimensional continua are worked out to illustrate the theory, in conjunction with the standard three-parameter model and the five-parameter model.
\end{abstract}

\section{Introduction}

Linear viscoelasticity, both in statics and dynamics, has often been considered a "simple" matter, since it's somewhat "similar" to linear elasticity. In fact, a well known elastic-viscoelastic correspondence principle [Flügge 1975] states that the two problems are formally equal when they are both transformed in the Laplace or Fourier domains. Thus, transformation is believed to be the best way to study viscoelastic problems, although often nontrivial antitransformations are needed when one desires to build the response time history [Narayanan and Beskos 1982].

There exist, of course, studies in which the system dynamics is analyzed in the time domain, for discrete or discretized systems, via finite or boundary elements methods (see, e.g., [Golla and Hughes 1985; Sim and Kwak 1988; McTavish and Hughes 1993; Schanz 1999; 2001; Hatada et al. 2000; Lewandowski et al. 2012; Syngellakis 2003; Baroudi et al. 2019]). An example of analysis which makes use of an the internal variable formulation is given in [Lewandowski et al. 2012]. All

\section{Communicated by Francesco dell'Isola.}

MSC2010: 74D05, 74H10, 74H40, 74H45, 74Q10.

Keywords: continuous viscoelastic metamodel, internal variables, linear dynamics, center manifold, multiple scales method, viscoelastic beam on viscoelastic Winkler soil, viscoelastic plate, viscoelastic Cauchy continuum. 
these studies, however, seem to not completely exploit the spectral characteristics of viscoelastic systems when damping, as usual, is small. In this case, indeed, the dynamics is made of a fast transient phase, and a successive steady-state phase, which takes place in a space of reduced dimensions, equal to those of the elastic system. This property was instead used by the authors in a recent paper [Luongo and D'Annibale 2017a], where a reduction method was proposed to contract the dimension of the discrete system to that of the associated elastic system. The procedure was based on the center manifold method, which is a common tool used in bifurcation analysis, designed to tackle nonlinear systems, and adapted in [Luongo and D'Annibale 2017a] to linear systems (similarly to what was done in [Shaw and Pierre 1991] in dealing with nonlinear normal modes).

The method discussed in [Luongo and D'Annibale 2017a] sheds light on another form of the correspondence principle which does not seem to have been explored yet, i.e., on a similarity holding not only in the transformed domains (where it exactly holds), but also in the time domain, although in the context of an asymptotic (and, therefore, approximate) theory. However, the procedure of [Luongo and D'Annibale 2017a] was limited to discrete systems, and did not seem, at first glance, to be straightforwardly extendible to infinite-dimensional systems. As a matter of fact, the center manifold theorem has only been proved for finitedimensional central subspaces (i.e., for a finite number of eigenvalues lying on the imaginary axis, or close to it). However, a favorable circumstance exists in the viscoelastic case, namely that the whole space of displacement and velocities is of interest. In other words, we do not have to worry about describing an infinitedimensional subspace in which a subset of displacements and velocities appear (as, for example, would be the case for a system undergoing an infinite number of buckling modes), but we have to take all the displacements and velocities which are admissible for the model. In this paper, we will prove (by a heuristic approach) that this circumstance still allows the use of the center manifold theorem.

The paper is organized as follows. In Section 2 a continuous viscoelastic metamodel using internal variables is formulated. In Section 3 both the center manifold and the multiple scales methods are applied to build a reduced system. In Sections 4, 5, and 6 sample systems, of increasing complexity, are worked out, and their reduced counterparts are derived. In Section 7 some conclusions are presented. Finally, in the Appendix an illustrative numerical example, concerning a homogenized microstructured viscoelastic one-dimensional continuum, is detailed.

\section{Continuous viscoelastic metamodel}

A dynamic linear metamodel of viscoelastic continuum is formulated. To this end a matrix notation is adopted, by denoting algebraic matrices by bold roman 

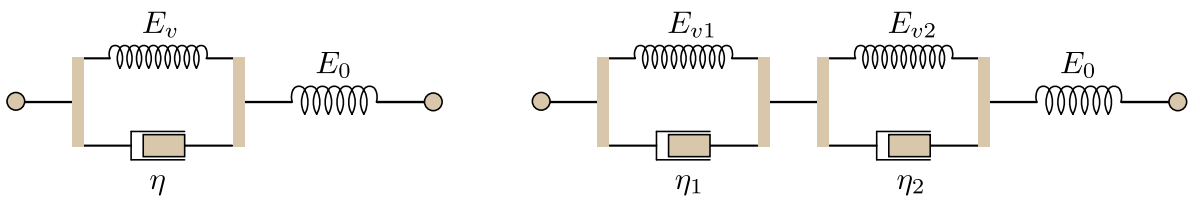

Figure 1. Rheological solid models: standard three-parameter (left) and five-parameter (right).

characters and formal matrices, made of linear differential operators, by bold calligraphic characters.

Kinematics and equilibrium of the system are governed by

$$
\begin{aligned}
\mathcal{D} u & =\boldsymbol{e}, \\
\mathcal{D}^{a} s & =f(x, t)-M \ddot{u},
\end{aligned}
$$

which are constrained by geometrical and mechanical boundary conditions. Here, $\boldsymbol{u}(\boldsymbol{x}, t), \boldsymbol{e}(\boldsymbol{x}, t), \boldsymbol{s}(\boldsymbol{x}, t)$ are column matrices collecting displacements, strains, and stress fields, respectively, and depending on position $\boldsymbol{x}$ and time $t ; \mathcal{D}$, and its adjoint $\mathcal{D}^{a}$, are the kinematic and equilibrium operators, collecting derivatives with respect to the coordinates $\boldsymbol{x} ; \boldsymbol{f}(\boldsymbol{x}, t)$ is the column matrix of the active forces; $\boldsymbol{M}$ is the inertia matrix; and the dot denotes differentiation with respect to the time $t$.

A class of viscoelastic constitutive laws is considered, which can be derived through kinematics and equilibrium and constitutive equations of rheological models. These latter are multiparameter solid models, made of Kelvin-Voigt (in-parallel spring/dashpot) elements, which are assembled in series to a spring device. Accordingly, the constitutive law reads

$$
\begin{aligned}
\boldsymbol{s} & =\boldsymbol{C}_{e}\left(\boldsymbol{e}-\boldsymbol{e}_{v}\right), \\
\boldsymbol{e}_{v} & =\boldsymbol{\Omega} \boldsymbol{\kappa}, \\
\dot{\boldsymbol{\kappa}} & =\boldsymbol{\Lambda}_{e} \boldsymbol{e}-\boldsymbol{\Lambda}_{R} \boldsymbol{\kappa} .
\end{aligned}
$$

Here, $\boldsymbol{e}_{v}$ is the viscous part of the strain, so that $\boldsymbol{e}-\boldsymbol{e}_{v}$ is the elastic part; stresses are assumed to be proportional to these latter parts by way of the squared elastic matrix $\boldsymbol{C}_{e}=\boldsymbol{C}_{e}^{T}$. The viscous strains, however, are linearly dependent on a generally larger number of internal variables $\boldsymbol{\kappa}$ [Moreau 1970; Maugin and Muschik 1990; Lemaitre and Chaboche 1990], to which they are connected via the rectangular matrix $\boldsymbol{\Omega}$, accounting for the topology of the underlying rheological model. For example, if reference is made to the standard three-parameter model (Figure 1, left), then $e_{v}$ and $\kappa$ coincide, being equal to the elongation $\varepsilon_{\mathrm{KV}}$ of the KelvinVoigt submodel; if, instead, the five-parameter model is adopted (Figure 1, right), $e_{v}=\kappa_{1}+\kappa_{2}$, with $\kappa_{i}(i=1,2)$ the elongation of the $i$-th Kelvin-Voigt submodel. 
Equation $(2)_{1}$ is the state law, while $(2)_{3}$ is the flow law, governing the evolution of the internal variables, here taken as linear; in it, $\boldsymbol{\Lambda}_{e}, \boldsymbol{\Lambda}_{R}$ are matrices of material constants, having dimension of the inverse of time, here referred to as relaxation matrices.

By combining (1) and (2) the following equations of motion, in state form, are derived:

$$
\left(\begin{array}{c}
\dot{\boldsymbol{u}} \\
\dot{\boldsymbol{v}} \\
\dot{\boldsymbol{\kappa}}
\end{array}\right)=\left[\begin{array}{ccc}
\mathbf{0} & \boldsymbol{I} & \mathbf{0} \\
-\boldsymbol{M}^{-1} \mathcal{K}_{e} & \mathbf{0} & \boldsymbol{M}^{-1} \mathcal{D}^{a} \boldsymbol{C}_{e} \boldsymbol{\Omega} \\
\boldsymbol{\Lambda}_{e} \mathcal{D} & \mathbf{0} & -\boldsymbol{\Lambda}_{R}
\end{array}\right]\left(\begin{array}{c}
\boldsymbol{u} \\
\boldsymbol{v} \\
\boldsymbol{\kappa}
\end{array}\right)+\left(\begin{array}{c}
\mathbf{0} \\
\boldsymbol{M}^{-1} \boldsymbol{f}(\boldsymbol{x}, t) \\
\mathbf{0}
\end{array}\right)
$$

where $\mathcal{K}_{e}:=\mathcal{D}^{a} C_{e} \mathcal{D}$ is the elastic stiffness operator. They are a set of equations in $N$ scalar displacement fields $\boldsymbol{u}, N$ scalar velocities $\boldsymbol{v}$, and $M$ internal variables $\boldsymbol{\kappa}$. System (3) must be integrated with boundary conditions and the initial conditions (assuming the system is initially at rest)

$$
\boldsymbol{u}(0)=\mathbf{0}, \quad \boldsymbol{v}(0)=\mathbf{0}, \quad \boldsymbol{\kappa}(0)=\mathbf{0} .
$$

\section{Subspace reduction}

The main hypothesis that all the viscous moduli of the structure are small is introduced so that the system is weakly damped. This smallness is accounted for by letting $\mathscr{O}\left(\left|\boldsymbol{\Lambda}_{\vartheta}\right|\right)=\epsilon^{-1}$, with $\epsilon$ a small perturbation parameter $(\vartheta=e, R)$. The existence of this parameter in the flow law makes the equations of motion singularly perturbed (since a small parameter affects the highest derivative [Fusco and Hale 1989; Nayfeh 2000]). It is easy to check, for example via a perturbation method [Luongo and D'Annibale 2017a], that such systems admit a well separated set of eigenvalues: (a) strongly damped real eigenvalues, on the order of $\epsilon^{-1}$, and (b) weakly damped complex conjugate eigenvalues, whose negative real part is of order $\epsilon$. The real eigenvalues are responsible for fast decaying motions and the complex eigenvalues for weakly decaying oscillatory motions. When $\epsilon$ decreases towards zero, the real eigenvalues move to the left side of the complex plane, making the decaying motion faster; the complex eigenvalues instead approach the imaginary axis, rendering the oscillatory motion more weakly decaying. The contrary, of course, occurs, when $\epsilon$ increases from zero. ${ }^{1}$

It is important to underline the fact that such a well separated spectrum can be recognized in viscoelastic structures when the order of magnitude of the relaxation time of the viscoelastic material is smaller than the natural periods of the structure.

\footnotetext{
${ }^{1}$ Note that this occurrence is completely different from the classical static phenomena of creep and relaxation tests, performed, e.g., on concrete and steel specimens. In those cases, indeed, damping is so strong that the real eigenvalues are closer to the imaginary axis than the complex eigenvalues, so that oscillations are fast damped, and the evolution is slow and quasistatic, driven by the real eigenvalues.
} 
This requirement cannot be met by most structural materials, since the behavior of a viscoelastic material with such a small relaxation time is close to that of a fluid. This demand could instead be satisfied, e.g., (i) in multilayered composites, (ii) in structures equipped with viscoelastic suspensions, or with fluid dampers (see, e.g., [Muscolino and Palmeri 2007]), or (iii) in homogenized microstructured viscoelastic systems and metamaterials, where specifically damping properties are designed (see, e.g., [Lekszycki et al. 1992; Manimala and Sun 2014; Del Vescovo and Giorgio 2014; Altenbach and Eremeyev 2015; Frazier and Hussein 2015; Giorgio et al. 2017; Lewińska et al. 2017; Barchiesi et al. 2019; Eugster et al. 2019]). In the Appendix, a one-dimensional example, i.e., a homogenized microstructured continuum, whose periodic microstructure is composed by standard three-parameter oscillators, sheds light on these aspects and permits discussing them numerically. All the sample systems, for which the subspace reduction will be carried out in the next sections, should be thought of as belonging to the categories mentioned above.

Coming back to slightly damped systems, it can be noticed that, if the transient dynamics related to the decaying eigenvalues is ignored, motions occur in a subspace spanned by the eigenvectors associated with the complex eigenvalues. This space, indeed, is much smaller than the original state space, so it is convenient to derive a reduced order model able to describe these dynamics. To construct the model, the space must be contracted without, of course, evaluating all the eigenvectors of the subspace; this is, indeed, possible, as will be illustrated soon.

Two alternative methods are worked out to achieve the goal, both of them borrowed from bifurcation theory, where they are commonly used to deal with nonlinear systems, namely (a) the center manifold method, and (b) the multiple scales method. The first one is a direct (but not trivial) generalization of the algorithm developed in [Luongo and D'Annibale 2017a] for discrete systems.

Center manifold method. According to the center manifold method, the eigenvalues are separated in the set of the stable eigenvalues (leftmost) and in the set of the central eigenvalues (close to the left side of imaginary axis). The associated eigenvectors span the stable and the central subspaces. The center manifold theorem [Guckenheimer and Holmes 1983; Wiggins 2003; Troger and Steindl 1991] assures that there exists an invariant manifold of the same dimensions as the central subspace $\pi$ that contains the origin, is tangent to the subspace $\pi$, and is attractive for the dynamics. Of course, since the system under study is linear, the manifold coincides with the subspace itself. However, differently from the hypotheses of the theorem, the center subspace is infinite-dimensional; in spite of this, the method is heuristically applied.

The Cartesian equations of the unknown subspace $\pi$ are

$$
\kappa=\mathcal{P} \boldsymbol{u}+\mathcal{Q} v
$$


where $\mathcal{P}$ and $\mathcal{Q}$ are unknown differential operators. If these operators are known, the first two equations of motion (3) 1,2 can be rewritten as

$$
\left(\begin{array}{c}
\dot{\boldsymbol{u}} \\
\dot{\boldsymbol{v}}
\end{array}\right)=\left[\begin{array}{cc}
\mathbf{0} & \boldsymbol{I} \\
-\boldsymbol{M}^{-1}\left(\mathcal{K}_{e}-\mathcal{D}^{a} \boldsymbol{C}_{e} \boldsymbol{\Omega} \mathcal{P}\right) & \boldsymbol{M}^{-1} \mathcal{D}^{a} \boldsymbol{C}_{e} \boldsymbol{\Omega \mathcal { Q }}
\end{array}\right]\left(\begin{array}{l}
\boldsymbol{u} \\
\boldsymbol{v}
\end{array}\right)+\left(\begin{array}{c}
\mathbf{0} \\
\boldsymbol{M}^{-1} \boldsymbol{f}
\end{array}\right)
$$

or

$$
M \ddot{\boldsymbol{u}}+\mathcal{B}_{r} \dot{\boldsymbol{u}}+\mathcal{K}_{r} \boldsymbol{u}=\boldsymbol{f}
$$

where

$$
\begin{aligned}
& \mathcal{B}_{r}:=-\mathcal{D}^{a} \boldsymbol{C}_{e} \boldsymbol{\Omega} \mathcal{Q}, \\
& \mathcal{K}_{r}:=\mathcal{K}_{e}-\mathcal{D}^{a} \boldsymbol{C}_{e} \boldsymbol{\Omega} \mathcal{P} .
\end{aligned}
$$

Equation (7) governs the linear dynamics of the system, reduced to the central subspace.

The task, therefore, is to determine $\mathcal{P}$ and $\mathcal{Q}$. This is accomplished by substituting (5) in the flow law $(3)_{3}$, with the help of $(3)_{1,2}$, and requiring it to be satisfied separately for independent $\boldsymbol{u}, \boldsymbol{v}$. It follows that

$$
\begin{aligned}
\left(-\mathcal{Q} M^{-1} \mathcal{K}_{e}+\mathcal{Q} M^{-1} \mathcal{D}^{a} \boldsymbol{C}_{e} \boldsymbol{\Omega} \mathcal{P}-\boldsymbol{\Lambda}_{e} \mathcal{D}+\boldsymbol{\Lambda}_{R} \mathcal{P}\right) \boldsymbol{u} & =\mathbf{0} \\
\left(\mathcal{P}+\mathcal{Q} \boldsymbol{M}^{-1} \mathcal{D}^{a} \boldsymbol{C}_{e} \boldsymbol{\Omega} \mathcal{Q}+\boldsymbol{\Lambda}_{R} \mathcal{Q}\right) \boldsymbol{v} & =\mathbf{0}
\end{aligned}
$$

In order to solve these nonlinear equations, a perturbation method is developed here. The viscous moduli are rescaled at the $\epsilon^{-1}$-order, so that $\boldsymbol{\Lambda}_{\vartheta} \rightarrow \epsilon^{-1} \boldsymbol{\Lambda}_{\vartheta}$ $(\vartheta=e, R)$ and, by multiplying the equations by $\epsilon$,

$$
\begin{array}{r}
{\left[\boldsymbol{\Lambda}_{R} \mathcal{P}-\boldsymbol{\Lambda}_{e} \mathcal{D}-\epsilon \mathcal{Q} M^{-1}\left(\mathcal{K}_{e}-\mathcal{D}^{a} C_{e} \boldsymbol{\Omega} \mathcal{P}\right)\right] \boldsymbol{u}=\mathbf{0},} \\
{\left[\boldsymbol{\Lambda}_{R} \mathcal{Q}+\epsilon\left(\mathcal{P}+\mathcal{Q} \boldsymbol{M}^{-1} \mathcal{D}^{a} \boldsymbol{C}_{e} \boldsymbol{\Omega} \mathcal{Q}\right)\right] \boldsymbol{v}=\mathbf{0} .}
\end{array}
$$

An inspection of these latter equations reveals that $\mathcal{P}(-\epsilon)=\mathcal{P}(\epsilon)$, while $\mathcal{Q}(-\epsilon)=$ $-\mathcal{Q}(\epsilon)$. Thus, the unknown matrices are expanded in series of even or odd powers of $\epsilon$, respectively:

$$
\begin{aligned}
& \mathcal{P}=\mathcal{P}_{0}+\epsilon^{2} \mathcal{P}_{2}+\cdots, \\
& \mathcal{Q}=\epsilon \mathcal{Q}_{1}+\epsilon^{3} \mathcal{Q}_{3}+\cdots,
\end{aligned}
$$

where $\mathcal{P}_{k}=\left.\frac{1}{k !} \frac{\mathrm{d}^{k} \mathcal{P}}{\mathrm{d} \epsilon^{k}}\right|_{\epsilon=0}$, with $k=0,2, \ldots$, and $\mathcal{Q}_{k}=\left.\frac{1}{k !} \frac{\mathrm{d}^{k} \mathcal{Q}}{\mathrm{d} \epsilon^{k}}\right|_{\epsilon=0}$, with $k=1,3, \ldots$ The following linear perturbation equations are then obtained:

$$
\begin{array}{rlrl}
\epsilon^{0}: & & \left(\boldsymbol{\Lambda}_{R} \mathcal{P}_{0}-\boldsymbol{\Lambda}_{e} \mathcal{D}\right) \boldsymbol{u} & =\mathbf{0}, \\
\epsilon^{1}: & \left(\boldsymbol{\Lambda}_{R} \mathcal{Q}_{1}+\mathcal{P}_{0}\right) \boldsymbol{v} & =\mathbf{0}, \\
\epsilon^{2}: & \left(\boldsymbol{\Lambda}_{R} \mathcal{P}_{2}-\mathcal{Q}_{1} \boldsymbol{M}^{-1}\left(\mathcal{K}_{e}-\mathcal{D}^{a} \boldsymbol{C}_{e} \boldsymbol{\Omega} \mathcal{P}_{0}\right)\right) \boldsymbol{u} & =\mathbf{0} .
\end{array}
$$


By solving them in cascade, it is found that

$$
\begin{aligned}
& \mathcal{P}_{0}=\boldsymbol{\Lambda}_{R}^{-1} \boldsymbol{\Lambda}_{e} \mathcal{D} \\
& \mathcal{Q}_{1}=-\boldsymbol{\Lambda}_{R}^{-2} \boldsymbol{\Lambda}_{e} \mathcal{D}, \\
& \mathcal{P}_{2}=-\boldsymbol{\Lambda}_{R}^{-3} \boldsymbol{\Lambda}_{e} \mathcal{D} \boldsymbol{M}^{-1}\left(\mathcal{K}_{e}-\mathcal{D}^{a} \boldsymbol{C}_{e} \boldsymbol{\Omega} \boldsymbol{\Lambda}_{R}^{-1} \boldsymbol{\Lambda}_{e} \mathcal{D}\right) .
\end{aligned}
$$

Finally, by reabsorbing the perturbation parameter, it follows that

$$
\begin{aligned}
& \mathcal{P}=\boldsymbol{\Lambda}_{R}^{-1} \boldsymbol{\Lambda}_{e} \mathcal{D}-\boldsymbol{\Lambda}_{R}^{-3} \boldsymbol{\Lambda}_{e} \mathcal{D} \boldsymbol{M}^{-1}\left(\mathcal{K}_{e}-\mathcal{D}^{a} \boldsymbol{C}_{e} \boldsymbol{\Omega} \boldsymbol{\Lambda}_{R}^{-1} \boldsymbol{\Lambda}_{e} \mathcal{D}\right)+\cdots, \\
& \mathcal{Q}=-\boldsymbol{\Lambda}_{R}^{-2} \boldsymbol{\Lambda}_{e} \mathcal{D}+\cdots
\end{aligned}
$$

Equations (14) are explicit relations that, once substituted in (8), permit us to build the reduced system (7). By truncating the analysis at the first nonzero term,

$$
\begin{aligned}
\mathcal{B}_{r} & :=\mathcal{D}^{a} \boldsymbol{C}_{e} \boldsymbol{\Omega} \boldsymbol{\Lambda}_{R}^{-2} \boldsymbol{\Lambda}_{e} \mathcal{D}, \\
\mathcal{K}_{r} & :=\mathcal{K}_{e}-\mathcal{D}^{a} \boldsymbol{C}_{e} \boldsymbol{\Omega} \boldsymbol{\Lambda}_{R}^{-1} \boldsymbol{\Lambda}_{e} \mathcal{D} .
\end{aligned}
$$

If, instead, an additional term is kept in the expansion of $\mathcal{P}$, an increment of stiffness must be added, so that $\mathcal{K}_{r}^{\mathrm{II}}:=\mathcal{K}_{r}+\Delta \mathcal{K}_{r}$, with

$$
\Delta \mathcal{K}_{r}=\mathcal{D}^{a} \boldsymbol{C}_{e} \boldsymbol{\Omega} \boldsymbol{\Lambda}_{R}^{-3} \boldsymbol{\Lambda}_{e} \mathcal{D} \boldsymbol{M}^{-1} \mathcal{K}_{r} .
$$

The first-order expressions (15) suggest two considerations. First, the damping matrix is a linear combination of the same differential operators which form the elastic (as well as the reduced) stiffness operator. However, due to the structure of the relaxation matrices, the coefficients of the linear combinations are different for the two matrices. Only under special circumstances does it occur that $\mathcal{B}_{r}$ and $\mathcal{K}_{r}$ are proportional; in general, the Rayleigh model of damping is not recovered.

As a second remark, the stiffness $\mathcal{K}_{r}$ has an important physical meaning. Indeed, if reference is made to a relaxation test, at the time $t=\infty$, it is $\dot{\boldsymbol{\kappa}}_{\infty}=0$; therefore, from the flow law (3) $)_{3}$, it follows that $\boldsymbol{\Lambda}_{R} \kappa_{\infty}=\boldsymbol{\Lambda}_{e} \mathcal{D} \boldsymbol{u}$. Hence, from (15)

$$
\mathcal{K}_{r} \boldsymbol{u}=\mathcal{D}^{a} \boldsymbol{C}_{e} \mathcal{D} \boldsymbol{u}-\mathcal{D}^{a} \boldsymbol{C}_{e} \boldsymbol{\Omega} \boldsymbol{\Lambda}_{R}^{-1} \boldsymbol{\Lambda}_{e} \mathcal{D} \boldsymbol{u}=\mathcal{D}^{a} \boldsymbol{C}_{e}\left(\boldsymbol{e}-\boldsymbol{\Omega} \boldsymbol{\kappa}_{\infty}\right)=\mathcal{D}^{a} \boldsymbol{s}_{\infty}
$$

where the elastic law (2) $)_{1}$ is used. By defining an elastic matrix at the equilibrium $\boldsymbol{C}_{e \infty}$, such that $\boldsymbol{C}_{e \infty} \boldsymbol{e}=\boldsymbol{s}_{\infty}$, it is concluded that

$$
\mathcal{K}_{r}=\mathcal{D}^{a} \boldsymbol{C}_{e \infty} \mathcal{D}=: \mathcal{K}_{e \infty},
$$

i.e., at the first order of the asymptotic expansion, the stiffness operator of the reduced system coincides with the elastic matrix, but with the elastic constants replaced by the equilibrium values of the relaxation moduli. This result is not surprising, since a weakly damped system is under consideration, for which the relaxation time is short and of the same order as the transient dynamics. Thus, 
when steady-state dynamics takes place on the central subspace, the relaxation moduli have attained their equilibrium values.

Multiple scales method. Now an alternative approach, based on the multiple scales method [Nayfeh 2000; Nayfeh and Mook 1979; Luongo and Zulli 2012a; 2012b; 2014; Luongo and D'Annibale 2013; 2017b; Luongo et al. 2016], is discussed. The flow law $(3)_{3}$ is rewritten with damping rescaled:

$$
\epsilon \dot{\boldsymbol{\kappa}}=\boldsymbol{\Lambda}_{e} \mathcal{D} \boldsymbol{u}-\boldsymbol{\Lambda}_{R} \boldsymbol{\kappa} .
$$

This is a singularly perturbed differential equation in which $\boldsymbol{u}$ (the active variable) is taken as known, and $\boldsymbol{\kappa}$ (the passive variable) as unknown. Although this equation is linear, with constant coefficients, the multiple scales method is applied to solve it, in order to get an asymptotic solution, useful to the reduction process.

First, several time scales $t_{k}:=\epsilon^{k} t$ with $k=0,1,2, \ldots$ are introduced and it assumed that $\boldsymbol{u}(\boldsymbol{x}, t)=\boldsymbol{u}\left(\boldsymbol{x}, t_{0}, t_{1}, \ldots\right)$ and $\boldsymbol{\kappa}(\boldsymbol{x}, t)=\boldsymbol{\kappa}\left(\boldsymbol{x}, t_{0}, t_{1}, \ldots\right)$; consequently, $\frac{\mathrm{d}}{\mathrm{d} t}=\partial_{t_{0}}+\epsilon \partial_{t_{1}}+\cdots$. Second, the unknown is expanded as

$$
\kappa=\kappa_{0}+\epsilon \kappa_{1}+\epsilon^{2} \kappa_{2}+\cdots
$$

where $\boldsymbol{\kappa}_{k}=\left.\frac{1}{k !} \frac{\mathrm{d}^{k} \boldsymbol{\kappa}_{k}}{\mathrm{~d} \epsilon^{k}}\right|_{\epsilon=0}$, with $k=0,1,2, \ldots$ The following perturbation equations are derived:

$$
\begin{array}{ll}
\epsilon^{0}: & \boldsymbol{\Lambda}_{R} \boldsymbol{\kappa}_{0}=\boldsymbol{\Lambda}_{e} \mathcal{D} \boldsymbol{u}, \\
\epsilon^{1}: & \boldsymbol{\Lambda}_{R} \boldsymbol{\kappa}_{1}=-\partial_{t_{0}} \boldsymbol{\kappa}_{0}, \\
\epsilon^{2}: & \boldsymbol{\Lambda}_{R} \boldsymbol{\kappa}_{2}=-\partial_{t_{0}} \boldsymbol{\kappa}_{1}-\partial_{t_{1}} \boldsymbol{\kappa}_{0} .
\end{array}
$$

The singular nature of the problem is encompassed by the fact that the generating, lower-order equation is not a differential equation in time. By solving the equations in cascade, it follows that

$$
\begin{aligned}
\boldsymbol{\kappa}_{0} & =\boldsymbol{\Lambda}_{R}^{-1} \boldsymbol{\Lambda}_{e} \mathcal{D} \boldsymbol{u}, \\
\boldsymbol{\kappa}_{1} & =-\boldsymbol{\Lambda}_{R}^{-2} \boldsymbol{\Lambda}_{e} \mathcal{D} \partial_{t_{0}} \boldsymbol{u}, \\
\boldsymbol{\kappa}_{2} & =\boldsymbol{\Lambda}_{R}^{-3} \boldsymbol{\Lambda}_{e} \mathcal{D} \partial_{t_{0}}^{2} \boldsymbol{u}-\boldsymbol{\Lambda}_{R}^{-2} \boldsymbol{\Lambda}_{e} \mathcal{D} \partial_{t_{1}} \boldsymbol{u} .
\end{aligned}
$$

By coming back to the series for $\kappa$, reabsorbing the perturbation parameter, and applying the "reconstitution principle" [Nayfeh 2000; Nayfeh and Mook 1979; Luongo and Paolone 1999], it is

$$
\boldsymbol{\kappa}=\boldsymbol{\Lambda}_{R}^{-1} \boldsymbol{\Lambda}_{e} \mathcal{D} \boldsymbol{u}-\boldsymbol{\Lambda}_{R}^{-2} \boldsymbol{\Lambda}_{e} \mathcal{D} \dot{\boldsymbol{u}}+\boldsymbol{\Lambda}_{R}^{-3} \boldsymbol{\Lambda}_{e} \mathcal{D} \ddot{\boldsymbol{u}}+\cdots,
$$

having taken into account that $\dot{\boldsymbol{u}}=\partial_{t_{0}} \boldsymbol{u}+\epsilon \partial_{t_{1}} \boldsymbol{u}+\cdots$ and $\ddot{\boldsymbol{u}}=\partial_{t_{0}}^{2} \boldsymbol{u}+\cdots$ and, consistently, having truncated the expansions. 
By substituting this latter expression in the equations of motion $(3)_{1,2}$ the reduced model is finally obtained as

$\left(\boldsymbol{M}-\mathcal{D}^{a} \boldsymbol{C}_{e} \boldsymbol{\Omega} \boldsymbol{\Lambda}_{R}^{-3} \boldsymbol{\Lambda}_{e} \mathcal{D}\right) \ddot{\boldsymbol{u}}+\left(\mathcal{D}^{a} \boldsymbol{C}_{e} \boldsymbol{\Omega} \boldsymbol{\Lambda}_{R}^{-2} \boldsymbol{\Lambda}_{e} \mathcal{D}\right) \dot{\boldsymbol{u}}+\left(\mathcal{K}_{e}-\mathcal{D}^{a} \boldsymbol{C}_{e} \boldsymbol{\Omega} \boldsymbol{\Lambda}_{R}^{-1} \boldsymbol{\Lambda}_{e} \mathcal{D}\right) \boldsymbol{u}=\boldsymbol{f}$.

In this equation, the stiffness and the damping operators are identical to those derived by the center manifold approach (see (15)). Moreover, a reduced mass (differential) operator appears, which was absent in the previous derivation. However, if the lower-order approximation (rendered homogeneous) is taken for $\ddot{\boldsymbol{u}}$, namely $\ddot{\boldsymbol{u}}=-\boldsymbol{M}^{-1} \mathcal{K}_{r} \boldsymbol{u}$, it is found that this extra term is consistent with the increment of stiffness (16). Therefore, the two approach are asymptotically equivalent.

The algorithm illustrated here better underlines the sequence of approximations, which is able to shed light on the physics of the systems. First, the internal variables are linked to the observable variables as $\dot{\boldsymbol{e}}_{v}=\mathbf{0}$, this approximation being sufficient to determine the reduced stiffness with the equilibrium relaxation moduli. Second, the resultant expression for $\boldsymbol{e}_{v}$ supplies the strain rate $\dot{\boldsymbol{e}}_{v}$, from which the damping is evaluated. Third, a better approximation for $\dot{\boldsymbol{e}}_{v}$ is computed that modifies the mass or, equivalently, the stiffness.

Throughout the following working examples, the second-order approximation is neglected.

\section{Viscoelastic Euler-Bernoulli beam on Winkler viscoelastic soil}

Model. The transverse motion of a shear-undeformable planar beam resting on viscoelastic soil is considered (Figure 2). Kinematics is described by

$$
\chi=u^{\prime \prime}, \quad \varepsilon=u
$$

where $u(s, t)$ is the deflection of the beam, $\chi(s, t)$ its curvature, and $\varepsilon(s, t)$ the elongation of soil, with $s$ the abscissa, $t$ the time, and a prime denoting $s$-differentiation.

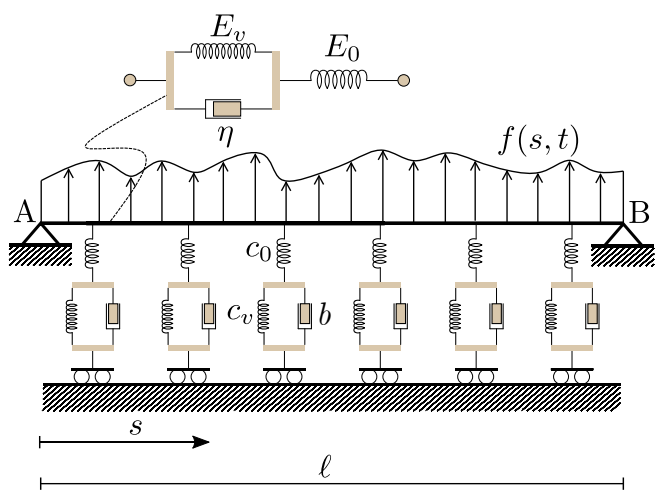

Figure 2. Planar viscoelastic beam on viscoelastic soil. 
Equilibrium entails

$$
M^{\prime \prime}=-r(s, t)-m \ddot{u}+f(s, t)
$$

where $M(s, t)$ is the bending moment, $r(s, t)$ the linear density of the soil reaction, $m$ the mass per unit length of the beam, and $f(s, t)$ the linear density of the external active forces.

The constitutive behavior of the beam and of the soil obeys the viscoelastic standard three-parameter model (Figure 1, left). The constitutive law for the beam is

$$
\begin{aligned}
& M=E_{0} I\left(\chi-\chi_{v}\right), \\
& \dot{\chi}_{v}=\frac{E_{0}}{\eta}\left(\chi-\chi_{v}\right)-\frac{E_{v}}{\eta} \chi_{v}
\end{aligned}
$$

where $E_{0}, E_{v}, \eta$ are material constants, $\chi_{v}$ is an internal variable having the meaning of viscous curvature, and $I$ is the cross-section inertia moment. The constitutive law for the soil is

$$
\begin{aligned}
r & =c_{0}\left(\varepsilon-\varepsilon_{v}\right), \\
\dot{\varepsilon}_{v} & =\frac{c_{0}}{b}\left(\varepsilon-\varepsilon_{v}\right)-\frac{c_{v}}{b} \varepsilon_{v}
\end{aligned}
$$

where $c_{0}, c_{v}, b$ are soil constants and $\varepsilon_{v}$ is an internal variable describing the viscous deformation of the soil. When all these equations are collected, and put in the state form, the viscoelastic problem reads

$$
\begin{aligned}
\dot{u} & =v, \\
m \dot{v}+E_{0} I\left(u^{\prime \prime \prime \prime}-\chi_{v}^{\prime \prime}\right)+c_{0}\left(u-\varepsilon_{v}\right) & =f(s, t), \\
\dot{\chi}_{v} & =\Lambda_{e b} \chi-\Lambda_{R b} \chi_{v}, \\
\dot{\varepsilon}_{v} & =\Lambda_{e s} \varepsilon-\Lambda_{R s} \varepsilon_{v}
\end{aligned}
$$

with $v$ the velocity, and the relaxation coefficients of beam and soil defined as

$$
\Lambda_{e b}:=\frac{E_{0}}{\eta}, \quad \Lambda_{R b}:=\frac{E_{0}+E_{v}}{\eta}, \quad \Lambda_{e s}:=\frac{c_{0}}{b}, \quad \Lambda_{R s}:=\frac{c_{0}+c_{v}}{b} .
$$

It is easy to check that (29) are of the type (3) with the identifications

$$
\begin{aligned}
& \boldsymbol{u}:=[u], \quad \boldsymbol{v}:=[v], \quad \boldsymbol{e}=\left[\begin{array}{l}
\chi \\
\varepsilon
\end{array}\right], \quad \boldsymbol{\kappa}=\left[\begin{array}{l}
\chi_{v} \\
\varepsilon_{v}
\end{array}\right], \\
& \mathcal{D}=\left[\begin{array}{c}
\partial_{s}^{2} \\
1
\end{array}\right], \quad \mathcal{D}^{a}=\left[\begin{array}{ll}
\partial_{s}^{2} & 1
\end{array}\right], \quad \boldsymbol{M}:=[m], \quad \boldsymbol{C}_{e}=\left[\begin{array}{cc}
E_{0} I & 0 \\
0 & c_{0}
\end{array}\right], \\
& \mathcal{K}_{e}=\left[E_{0} I \partial_{s}^{4}+c_{0}\right], \boldsymbol{\Lambda}_{e}=\left[\begin{array}{cc}
\Lambda_{e b} & 0 \\
0 & \Lambda_{e s}
\end{array}\right], \boldsymbol{\Lambda}_{R}=\left[\begin{array}{cc}
\Lambda_{R b} & 0 \\
0 & \Lambda_{R s}
\end{array}\right], \quad \boldsymbol{\Omega}=\left[\begin{array}{ll}
1 & 0 \\
0 & 1
\end{array}\right]
\end{aligned}
$$

where $\partial_{s}^{k}:=\frac{\partial^{k}}{\partial s^{k}}$. 
Equations (29) must be constrained by boundary conditions. For example, if the beam is simply supported at the ends A, B, boundary conditions read

$$
u_{H}=0, \quad E_{0} I\left(u_{H}^{\prime \prime}-\chi_{v H}\right)=0, \quad H=\mathrm{A}, \mathrm{B} .
$$

Reduced system. The reduction process requires linearly linking the internal variables to the observable variables via unknown differential operators $P_{\vartheta}$ and $Q_{\vartheta}$ $(\vartheta=b, s)($ remember $(5))$ :

$$
\left(\begin{array}{l}
\chi_{v} \\
\varepsilon_{v}
\end{array}\right)=\left[\begin{array}{l}
P_{b} \\
P_{s}
\end{array}\right] u+\left[\begin{array}{l}
Q_{b} \\
Q_{s}
\end{array}\right] v=\mathcal{P} \boldsymbol{u}+\mathcal{Q} v .
$$

By using (14) and the definitions (31), truncated at the first term, it is found that

$$
\mathcal{P}=\left[\begin{array}{c}
\left(\Lambda_{e b} / \Lambda_{R b}\right) \partial_{s}^{2} \\
\Lambda_{e s} / \Lambda_{R s}
\end{array}\right], \quad \mathcal{Q}=-\left[\begin{array}{c}
\left(\Lambda_{e b} / \Lambda_{R b}^{2}\right) \partial_{s}^{2} \\
\Lambda_{e s} / \Lambda_{R s}^{2}
\end{array}\right]
$$

so that the operators are mixed, differential-algebraic. With these, from (8), or (15), the stiffness and damping of the reduced system are found, namely

$$
\begin{aligned}
\mathcal{B}_{r} & =\left[E_{0} I \frac{\Lambda_{e b}}{\Lambda_{R b}^{2}} \partial_{s}^{4}+c_{0} \frac{\Lambda_{e s}}{\Lambda_{R s}^{2}}\right], \\
\mathcal{K}_{r} & =\left[E_{0} I\left(1-\frac{\Lambda_{e b}}{\Lambda_{R b}}\right) \partial_{s}^{4}+c_{0}\left(1-\frac{\Lambda_{e s}}{\Lambda_{R s}}\right)\right] .
\end{aligned}
$$

Finally, the reduced equation of motion (7), in terms of only observable variables, reads

$$
m \ddot{u}+\alpha I \dot{u}^{\prime \prime \prime \prime}+b^{\star} \dot{u}+E_{\infty} I u^{\prime \prime \prime \prime \prime}+c_{\infty} u=f
$$

where, by accounting for the definitions (30), the following viscoelastic coefficients have been introduced:

$$
\alpha:=\eta\left(\frac{E_{0}}{E_{0}+E_{v}}\right)^{2}, \quad b^{\star}:=b\left(\frac{c_{0}}{c_{0}+c_{v}}\right)^{2}, \quad E_{\infty}:=\frac{E_{0} E_{v}}{E_{0}+E_{v}}, \quad c_{\infty}:=\frac{c_{0} c_{v}}{c_{0}+c_{v}} .
$$

Note that this model describes an internally and externally damped beam on a Winkler elastic soil; in particular, $\alpha$ is the internal damping coefficient, depending on the elastic and viscous properties of the beam; $b^{\star}$ is the external damping coefficient, which depends on the elastic and viscous properties of the soil; and $E_{\infty}, c_{\infty}$ are the relaxation moduli at the equilibrium. It is worth noticing that, in this model, damping is not proportional to the stiffness.

The internal damping of the beam. When the beam is in the air (i.e., not resting on soil, so $c_{0}=c_{v}=0$ ) the reduced model (36), in the absence of external forces, becomes

$$
m \ddot{u}+\eta\left(\frac{E_{0}}{E_{0}+E_{v}}\right)^{2} I \dot{u}^{\prime \prime \prime \prime \prime}+E_{\infty} I u^{\prime \prime \prime \prime \prime}=0 .
$$


Let $\phi_{j}(s)$ be the $j$-th natural mode of the elastic beam, of frequency $\omega_{j}$, satisfying

$$
E_{\infty} I \phi_{j}^{\prime \prime \prime \prime}-m \omega_{j}^{2} \phi_{j}=0
$$

with the relevant boundary conditions. Since damping is now of proportional type, $\phi_{j}$ is also an eigenvector for the viscoelastic beam. Thus, by substituting $u(s, t)=x_{j}(t) \phi_{j}(s)$ in the equation of motion, and accounting for (39), an ordinary differential equation for the modal amplitude $x_{j}(t)$ is obtained, namely

$$
\ddot{x}_{j}+\eta \omega_{j}^{2} \frac{E_{\infty}}{E_{v}^{2}} \dot{x}_{j}+\omega_{j}^{2} x_{j}=0 .
$$

With the usual definition of damping factor, and by using $(37)_{3}$ and $(30)_{2}$, it is found that

$$
\xi_{j}=\frac{1}{2} \eta \frac{E_{\infty}}{E_{v}^{2}} \omega_{j} \equiv \frac{1}{2} \frac{E_{0}}{E_{v}} \frac{\omega_{j}}{\Lambda_{R}}
$$

where $\Lambda_{R} \equiv \Lambda_{R b}$ and which, consistently with the Rayleigh model of damping (proportional to the stiffness), is proportional to the undamped natural frequency. This last expression is believed to be useful in damping modeling and design of beams.

\section{Viscoelastic Kirchhoff plate}

Model. The transverse vibrations of a thin viscoelastic plate, modeled according to Kirchhoff's theory, are considered (see Figure 3).

Kinematics is described by

$$
\left(\begin{array}{l}
\chi_{x} \\
\chi_{y} \\
\chi_{x y}
\end{array}\right)=\left[\begin{array}{c}
\partial_{x x}^{2} \\
\partial_{y y}^{2} \\
2 \partial_{x y}^{2}
\end{array}\right](u)
$$

where $u(\boldsymbol{x}, t)$ is the transverse deflection, with $\boldsymbol{x}=x \boldsymbol{a}_{x}+y \boldsymbol{a}_{y}$ the position; $\chi_{x}(\boldsymbol{x}, t)$, $\chi_{y}(\boldsymbol{x}, t)$ are flexural curvatures and $\chi_{x y}(\boldsymbol{x}, t)$ the torsional curvature; and finally

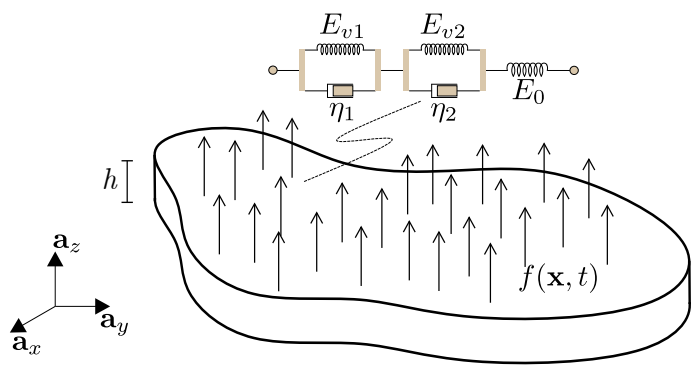

Figure 3. Viscoelastic Kirchhoff plate. 
$\partial_{\vartheta_{1} \cdots \vartheta_{k}}^{k}\left(\vartheta_{i}=x, y\right)$ denotes the partial derivative of order $k$ with respect the homonymous variables.

Equilibrium is ruled by

$$
\left[\partial_{x x}^{2}, \partial_{y y}^{2}, 2 \partial_{x y}^{2}\right]\left(\begin{array}{c}
M_{x} \\
M_{y} \\
M_{x y}
\end{array}\right)=f-\rho \ddot{u}
$$

where $M_{x}, M_{y}$ are bending moments and $M_{x y}$ the torsional moment; moreover, $f(\boldsymbol{x}, t)$ is the surface load density, and $\rho$ the surface mass density.

The constitutive law of the plate is assumed to be governed by the five-parameter model, which is made of one spring $E_{0}$, placed in series with two Kelvin-Voigt systems of constants $E_{v i}, \eta_{i}(i=1,2)$ (Figure 1, right). Moreover, it is also admitted that all the stress components could evolve by the same law with strain components (thus entailing that the deviatoric and spherical stresses of the underlying three-dimensional model have the same viscoelastic evolution). Under these assumptions, the elastic law is

$$
\left(\begin{array}{c}
M_{x} \\
M_{y} \\
M_{x y}
\end{array}\right)=D_{0}\left[\begin{array}{ccc}
1 & v & 0 \\
v & 1 & 0 \\
0 & 0 & (1-v) / 2
\end{array}\right]\left(\begin{array}{c}
\chi_{x}-\kappa_{x 1}-\kappa_{x 2} \\
\chi_{y}-\kappa_{y 1}-\kappa_{y 2} \\
\chi_{x y}-\kappa_{x y 1}-\kappa_{x y 2}
\end{array}\right)
$$

where $\kappa_{\vartheta i}(\vartheta=x, y, x y$ and $i=1,2)$ are the viscous curvatures, relevant to the $i$-th subcomponent of the rheological model, and $D_{0}:=\frac{1}{12} h^{3} E_{0} /\left(1-v^{2}\right)$ is the bending stiffness, with $E_{0}, v$ the Young modulus and the Poisson ratio, and $h$ the plate thickness.

The flow laws, governing the evolution of the internal variables $\kappa_{\vartheta i}$, are

$$
\begin{array}{r}
\left(\begin{array}{l}
\dot{\kappa}_{\vartheta 1} \\
\dot{\kappa}_{\vartheta 2}
\end{array}\right)=\left[\begin{array}{l}
E_{0} / \eta_{1} \\
E_{0} / \eta_{2}
\end{array}\right]\left(\chi_{\vartheta}\right)-\left[\begin{array}{cc}
\left(E_{0}+E_{v 1}\right) / \eta_{1} & E_{0} / \eta_{1} \\
E_{0} / \eta_{2} & \left(E_{0}+E_{v 2}\right) / \eta_{2}
\end{array}\right] \\
\left.\vartheta \begin{array}{l}
\kappa_{\vartheta 1} \\
\kappa_{\vartheta 2}
\end{array}\right), \\
\vartheta=x, y, x y .
\end{array}
$$

All these equations are in the form (1)-(2) and, therefore, implicitly define (i) the differential operators $\mathcal{D}, \mathcal{D}^{a}$ in (42)-(43), (ii) the topological matrix $\boldsymbol{\Omega}$, linking the internal variables, i.e., the viscous curvatures $\kappa_{\vartheta i}$, to the viscous strains (remember $(2)_{2}$ ), and (iii) the elastic matrix $\boldsymbol{C}_{\boldsymbol{e}}$. They read

$$
\begin{array}{rlrl}
\mathcal{D}:=\left[\begin{array}{c}
\partial_{x x}^{2} \\
\partial_{y y}^{2} \\
2 \partial_{x y}^{2}
\end{array}\right], & & \mathcal{D}^{a}:=\left[\partial_{x x}^{2}, \partial_{y y}^{2}, 2 \partial_{x y}^{2}\right], \\
\boldsymbol{\Omega}:=\left[\begin{array}{llllll}
1 & 1 & 0 & 0 & 0 & 0 \\
0 & 0 & 1 & 1 & 0 & 0 \\
0 & 0 & 0 & 0 & 1 & 1
\end{array}\right], & \boldsymbol{C}_{e}:=D_{0}\left[\begin{array}{ccc}
1 & v & 0 \\
v & 1 & 0 \\
0 & 0 & (1-v) / 2
\end{array}\right] .
\end{array}
$$


Moreover, from (45) the definition of $\boldsymbol{\Lambda}_{e}, \boldsymbol{\Lambda}_{R}$ follows, namely

$$
\begin{aligned}
\boldsymbol{\Lambda}_{e}:= & {\left[\begin{array}{ccc}
E_{0} / \eta_{1} & 0 & 0 \\
E_{0} / \eta_{2} & 0 & 0 \\
0 & E_{0} / \eta_{1} & 0 \\
0 & E_{0} / \eta_{2} & 0 \\
0 & 0 & E_{0} / \eta_{1} \\
0 & 0 & E_{0} / \eta_{2}
\end{array}\right], } \\
\boldsymbol{\Lambda}_{R}:= & {\left[\begin{array}{cccccc}
F_{1} & E_{0} / \eta_{1} & 0 & 0 & 0 & 0 \\
E_{0} / \eta_{2} & F_{2} & 0 & 0 & 0 & 0 \\
0 & 0 & F_{1} & E_{0} / \eta_{1} & 0 & 0 \\
0 & 0 & E_{0} / \eta_{2} & F_{2} & 0 & 0 \\
0 & 0 & 0 & 0 & F_{1} & E_{0} / \eta_{1} \\
0 & 0 & 0 & 0 & E_{0} / \eta_{2} & F_{2}
\end{array}\right] }
\end{aligned}
$$

where $F_{j}=\left(E_{0}+E_{v j}\right) / \eta_{j}(j=1,2)$. The equations of motion, when rearranged in the state form (3), are

$$
\dot{w}=\mathcal{A} w+f
$$

where the definitions

$$
\begin{aligned}
& \boldsymbol{w}:=\left(\begin{array}{llllllll}
u & v & \kappa_{x 1} & \kappa_{x 2} & \kappa_{y 1} & \kappa_{y 2} & \kappa_{x y 1} & \kappa_{x y 2}
\end{array}\right)^{T}, \\
& \boldsymbol{f}:=\left(\begin{array}{llllllll}
0 & f / \rho & 0 & 0 & 0 & 0 & 0 & 0
\end{array}\right)^{T}, \\
& \mathcal{A}:=\left[\begin{array}{ccc}
\mathbf{0} & \boldsymbol{I} & \mathbf{0} \\
-(1 / \rho) \mathcal{K}_{e} & \mathbf{0} & (1 / \rho) \mathcal{D}^{a} \boldsymbol{C}_{e} \boldsymbol{\Omega} \\
\boldsymbol{\Lambda}_{e} \mathcal{D} & \mathbf{0} & -\boldsymbol{\Lambda}_{R}
\end{array}\right]
\end{aligned}
$$

hold and in which

$$
\begin{aligned}
\mathcal{K}_{e}:= & D_{0} \nabla^{4} \\
\mathcal{D}^{a} \boldsymbol{C}_{e} \boldsymbol{\Omega}= & D_{0}\left[\partial_{x x}^{2}+v \partial_{y y}^{2} \partial_{x x}^{2}+v \partial_{y y}^{2} \partial_{y y}^{2}+v \partial_{x x}^{2} \partial_{y y}^{2}+v \partial_{x x}^{2}(1-v) \partial_{x y}^{2}(1-v) \partial_{x y}^{2}\right] \\
\boldsymbol{\Lambda}_{e} \mathcal{D}: & :\left(\begin{array}{c}
\left(E_{0} / \eta_{1}\right) \partial_{x x}^{2} \\
\left(E_{0} / \eta_{2}\right) \partial_{x x}^{2} \\
\left(E_{0} / \eta_{1}\right) \partial_{y y}^{2} \\
\left(E_{0} / \eta_{2}\right) \partial_{y y}^{2} \\
2\left(E_{0} / \eta_{1}\right) \partial_{x y}^{2} \\
2\left(E_{0} / \eta_{2}\right) \partial_{x y}^{2}
\end{array}\right) .
\end{aligned}
$$

The dynamics is, therefore, governed by a system of partial differential equations in two observable variables (displacement and velocity) and six internal variables (two viscous components for each of the three curvatures). 
Reduced system. Aimed at reducing the system to the sole observable variables, the internal variables are linked to them via unknown differential operators $\mathcal{P}, \mathcal{Q}$, i.e.,

$$
\left(\begin{array}{c}
\kappa_{x 1} \\
\kappa_{x 2} \\
\kappa_{y 1} \\
\kappa_{y 2} \\
\kappa_{x y 1} \\
\kappa_{x y 2}
\end{array}\right)=\left[\begin{array}{c}
P_{x 1} \\
P_{x 2} \\
P_{y 1} \\
P_{y 2} \\
P_{x y 1} \\
P_{x y 2}
\end{array}\right] u+\left[\begin{array}{l}
Q_{x 1} \\
Q_{x 2} \\
Q_{y 1} \\
Q_{y 2} \\
Q_{x y 1} \\
Q_{x y 2}
\end{array}\right] v=\mathcal{P} \boldsymbol{u}+\mathcal{Q} \boldsymbol{v} .
$$

With the definitions (50) and by using the first-term approximation in (14),

$$
\begin{aligned}
\mathcal{P}=\frac{E_{0}}{E_{0} E_{v 1}+E_{v 1} E_{v 2}+E_{0} E_{v 2}}\left[\begin{array}{c}
E_{v 2} \partial_{x x}^{2} \\
E_{v 1} \partial_{x x}^{2} \\
E_{v 2} \partial_{y y}^{2} \\
E_{v 1} \partial_{y y}^{2} \\
2 E_{v 2} \partial_{x y}^{2} \\
2 E_{v 1} \partial_{x y}^{2}
\end{array}\right], \\
\mathcal{Q}=\frac{E_{0}}{\left(E_{0} E_{v 1}+E_{v 1} E_{v 2}+E_{0} E_{v 2}\right)^{2}}\left[\begin{array}{c}
-\left(E_{0} E_{v 2} \eta_{1}+E_{v 2}^{2} \eta_{1}-E_{0} E_{v 1} \eta_{2}\right) \partial_{x x}^{2} \\
\left(E_{0} E_{v 2} \eta_{1}-E_{v 1}^{2} \eta_{2}-E_{0} E_{v 1} \eta_{2}\right) \partial_{x x}^{2} \\
-\left(E_{0} E_{v 2} \eta_{1}+E_{v 2}^{2} \eta_{1}-E_{0} E_{v 1} \eta_{2}\right) \partial_{y y}^{2} \\
\left(E_{0} E_{v 2} \eta_{1}-E_{v 1}^{2} \eta_{2}-E_{0} E_{v 1} \eta_{2}\right) \partial_{y y}^{2} \\
-2\left(E_{0} E_{v 2} \eta_{1}+E_{v 2}^{2} \eta_{1}-E_{0} E_{v 1} \eta_{2}\right) \partial_{x y}^{2} \\
2\left(E_{0} E_{v 2} \eta_{1}-E_{v 1}^{2} \eta_{2}-E_{0} E_{v 1} \eta_{2}\right) \partial_{x y}^{2}
\end{array}\right]
\end{aligned}
$$

so that the operators (8) of the reduced system are

$$
\mathcal{B}_{r}:=\alpha \nabla^{4}, \quad \mathcal{K}_{r}:=D_{\infty} \nabla^{4}
$$

where the definitions

$$
\alpha:=D_{0} \frac{E_{0}\left(E_{v 2}^{2} \eta_{1}+E_{v 1}^{2} \eta_{2}\right)}{\left(E_{0} E_{v 1}+E_{v 1} E_{v 2}+E_{0} E_{v 2}\right)^{2}}, \quad D_{\infty}:=\frac{h^{3}}{12} \frac{E_{\infty}}{1-v^{2}}
$$

hold, $D_{\infty}$ being the stiffness of the plate at $t=\infty$ with

$$
E_{\infty}:=\frac{E_{0} E_{v 1} E_{v 2}}{E_{0} E_{v 1}+E_{v 1} E_{v 2}+E_{0} E_{v 2}}
$$

the stiffness of the three in-series springs of the five-parameter model.

By summarizing, the reduced model is governed by

$$
D_{\infty} \nabla^{4} u+\alpha \nabla^{4} \dot{u}+\rho \ddot{u}=f .
$$

In this case the damping, governed by the coefficient $\alpha$, is found to be of proportional type, due to the hypotheses made on the constitutive law. 


\section{The Cauchy viscoelastic continuum}

Model. An isotropic Cauchy continuum is considered. The vector of displacements, at point $\boldsymbol{x}$ and time $t$, is denoted by $\boldsymbol{u}(\boldsymbol{x}, t)$, the infinitesimal strain tensor by $\boldsymbol{E}(\boldsymbol{x}, t)$, and the stress tensor by $\boldsymbol{T}(\boldsymbol{x}, t)$. Then the tensors are decomposed in their spherical and deviatoric parts, namely $\boldsymbol{E}=\frac{1}{3} \delta \boldsymbol{I}+\boldsymbol{E}^{\star}$ and $\boldsymbol{T}=\theta \boldsymbol{I}+\boldsymbol{T}^{\star}$, where $\delta:=\operatorname{tr} \boldsymbol{E}$ is the volumetric strain, $\theta=\operatorname{tr} \boldsymbol{T}$ is the hydrostatic stress, $\boldsymbol{I}$ is the identity tensor, and a star denotes the deviatoric part.

Kinematics states that

$$
\delta=\nabla \cdot \boldsymbol{u}, \quad \boldsymbol{E}^{\star}=\frac{1}{2}\left[\nabla \boldsymbol{u}+(\nabla \boldsymbol{u})^{T}\right]-\frac{1}{3}(\boldsymbol{I} \nabla \cdot \boldsymbol{u}) .
$$

Equilibrium calls for

$$
\nabla \cdot\left(\theta \boldsymbol{I}+\boldsymbol{T}^{\star}\right)-\rho \ddot{\boldsymbol{u}}+\boldsymbol{b}=\mathbf{0}
$$

where $\rho$ is the mass density and $\boldsymbol{b}$ the bulk forces.

It is assumed that the body is linear viscoelastic in the deviatoric part, while it is elastic in the spherical part. By adopting the standard three-parameter model, the constitutive law is written as

$$
\begin{aligned}
\boldsymbol{T}^{\star} & =2 \mu\left(\boldsymbol{E}^{\star}-\boldsymbol{E}_{v}^{\star}\right), \\
\dot{\boldsymbol{E}}_{v}^{\star} & =\Lambda_{e} \boldsymbol{E}^{\star}-\Lambda_{R} \boldsymbol{E}_{v}^{\star}, \\
\theta & =K \delta
\end{aligned}
$$

where $\boldsymbol{E}_{v}^{\star}$ is the viscous part and $\boldsymbol{E}^{\star}-\boldsymbol{E}_{v}^{\star}$ the elastic part of the deviatoric strain tensor; $\mu$ and $K:=\lambda+\frac{2}{3} \mu$ are elastic constants, namely the Lamé constants $\lambda, \mu$ and the bulk modulus $K$; and finally, $\Lambda_{e}, \Lambda_{R}$ are relaxation constants.

By arranging the previous equations in the state form (accounting for $\nabla \cdot \nabla \boldsymbol{u}=$ $\nabla^{2} \boldsymbol{u}, \nabla \cdot(\nabla \boldsymbol{u})^{T}=\nabla(\nabla \cdot \boldsymbol{u})$, and $\left.\nabla \cdot(\boldsymbol{I} \nabla \cdot \boldsymbol{u})=\nabla(\nabla \cdot \boldsymbol{u})\right)$, it is

$$
\begin{aligned}
& \dot{\boldsymbol{u}}=\boldsymbol{v}, \\
& \mu \nabla^{2} \boldsymbol{u}+(\mu+\lambda) \nabla(\nabla \cdot \boldsymbol{u})-2 \mu \nabla \cdot \boldsymbol{E}_{v}^{\star}-\rho \dot{\boldsymbol{v}}+\boldsymbol{b}=\mathbf{0}, \\
& \dot{\boldsymbol{E}}_{v}^{\star}=\Lambda_{e}\left[\frac{1}{2} \nabla \boldsymbol{u}+\frac{1}{2}(\nabla \boldsymbol{u})^{T}-\frac{1}{3} \boldsymbol{I} \nabla \cdot \boldsymbol{u}\right]-\Lambda_{R} \boldsymbol{E}_{v}^{\star} .
\end{aligned}
$$

These equations are of the general form (3), although here a different notation is used (vector form, instead of matrix form). When $\boldsymbol{E}_{v}^{\star} \equiv \mathbf{0}$ and the flow law is suppressed, they reduce to the well known Navier equations for the elastic continuum.

Reduced system. To reduce the system to the central subspace, the procedure illustrated for a general system is repeated, by using the vector notation. First, the internal variables are expressed as a linear combination of the displacement and velocity:

$$
\boldsymbol{E}_{v}^{\star}=\mathcal{P} \boldsymbol{u}+\mathcal{Q} \boldsymbol{v}
$$


where $\mathcal{P}, \mathcal{Q}$ are unknown linear differential operators. By differentiating this latter equation with respect to time, using $(60)_{1,2}$ (rendered homogeneous) to eliminate $\dot{\boldsymbol{u}}, \dot{\boldsymbol{v}}$, and similarly using (61) to eliminate $\boldsymbol{E}_{v}^{\star},(60)_{3}$ reads

$$
\begin{aligned}
\mathcal{P} \boldsymbol{v}+\frac{1}{\rho} \mathcal{Q}\left[\mu \nabla^{2} \boldsymbol{u}+(\right. & (\mu+\lambda) \nabla(\nabla \cdot \boldsymbol{u})-2 \mu \nabla \cdot(\mathcal{P} \boldsymbol{u}+\mathcal{Q} \boldsymbol{v})] \\
& =\Lambda_{e}\left[\frac{1}{2} \nabla \boldsymbol{u}+\frac{1}{2}(\nabla \boldsymbol{u})^{T}-\frac{1}{3} \boldsymbol{I} \nabla \cdot \boldsymbol{u}\right]-\Lambda_{R}(\mathcal{P} \boldsymbol{u}+\mathcal{Q} \boldsymbol{v}) .
\end{aligned}
$$

By requiring it holds for any $\boldsymbol{u}, \boldsymbol{v}$, it follows that

$$
\begin{array}{r}
\frac{1}{\rho} \mathcal{Q}\left[\mu \nabla^{2} \boldsymbol{u}+(\mu+\lambda) \nabla(\nabla \cdot \boldsymbol{u})-2 \mu \nabla \cdot(\mathcal{P} \boldsymbol{u})\right]-\Lambda_{e}\left[\frac{1}{2} \nabla \boldsymbol{u}+\frac{1}{2}(\nabla \boldsymbol{u})^{T}-\frac{1}{3} \boldsymbol{I} \nabla \cdot \boldsymbol{u}\right] \\
+\Lambda_{R}(\mathcal{P} \boldsymbol{u})=\mathbf{0}, \\
\mathcal{P} \boldsymbol{v}-\frac{2 \mu}{\rho} \mathcal{Q}[\nabla \cdot(\mathcal{Q} \boldsymbol{v})]+\Lambda_{R} \mathcal{Q} \boldsymbol{v}=\mathbf{0} .
\end{array}
$$

Then, by rescaling $\Lambda_{\vartheta} \rightarrow \epsilon^{-1} \Lambda_{\vartheta}(\vartheta=e, R)$ and expanding the operators as $\mathcal{P}=$ $\mathcal{P}_{0}+\mathcal{O}(\epsilon)$ and $\mathcal{Q}=\epsilon \mathcal{Q}_{1}+\mathcal{O}\left(\epsilon^{2}\right)$, the leading perturbation equations

$$
\begin{aligned}
-\Lambda_{e}\left[\frac{1}{2} \nabla \boldsymbol{u}+\frac{1}{2}(\nabla \boldsymbol{u})^{T}-\frac{1}{3} \boldsymbol{I} \nabla \cdot \boldsymbol{u}\right]+\Lambda_{R}\left(\mathcal{P}_{0} \boldsymbol{u}\right) & =\mathbf{0}, \\
\mathcal{P}_{0} \boldsymbol{v}+\Lambda_{R} \mathcal{Q}_{1} \boldsymbol{v} & =\mathbf{0}
\end{aligned}
$$

are obtained, whose solution is

$$
\begin{aligned}
& \mathcal{P}_{0} \boldsymbol{u}=\frac{1}{2} \frac{\Lambda_{e}}{\Lambda_{R}}\left[\nabla \boldsymbol{u}+(\nabla \boldsymbol{u})^{T}\right]-\frac{1}{3} \frac{\Lambda_{e}}{\Lambda_{R}} \boldsymbol{I} \nabla \cdot \boldsymbol{u}, \\
& \mathcal{Q}_{1} \boldsymbol{v}=-\frac{1}{2} \frac{\Lambda_{e}}{\Lambda_{R}^{2}}\left[\nabla \boldsymbol{v}+(\nabla \boldsymbol{v})^{T}\right]+\frac{1}{3} \frac{\Lambda_{e}}{\Lambda_{R}^{2}} \boldsymbol{I} \nabla \cdot \boldsymbol{v} .
\end{aligned}
$$

Therefore, from (61), at the leading order, it is found that

$$
\boldsymbol{E}_{v}^{\star}=\frac{1}{2} \frac{\Lambda_{e}}{\Lambda_{R}}\left[\nabla \boldsymbol{u}+(\nabla \boldsymbol{u})^{T}\right]-\frac{1}{3} \frac{\Lambda_{e}}{\Lambda_{R}} \boldsymbol{I} \nabla \cdot \boldsymbol{u}-\frac{1}{2} \frac{\Lambda_{e}}{\Lambda_{R}^{2}}\left(\nabla \boldsymbol{v}+\nabla^{T} \boldsymbol{v}\right)+\frac{1}{3} \frac{\Lambda_{e}}{\Lambda_{R}^{2}} \boldsymbol{I} \nabla \cdot \boldsymbol{v},
$$

which permits us to write the balance equations $(60)_{1,2}$ in terms of displacements only:

$$
\begin{array}{r}
\mu_{\infty} \nabla^{2} \boldsymbol{u}+\left[\frac{\mu_{\infty}\left(\Lambda_{e}-3 \Lambda_{R}\right)}{3\left(\Lambda_{e}-\Lambda_{R}\right)}+\lambda\right] \nabla(\nabla \cdot \boldsymbol{u})-\frac{\mu_{\infty} \Lambda_{e}}{\Lambda_{R}\left(\Lambda_{e}-\Lambda_{R}\right)}\left(\nabla^{2} \dot{\boldsymbol{u}}+\frac{1}{3} \nabla(\nabla \cdot \dot{\boldsymbol{u}})\right) \\
-\rho \ddot{\boldsymbol{u}}+\boldsymbol{b}=\mathbf{0}
\end{array}
$$

where $\mu_{\infty}:=\mu\left(1-\Lambda_{e} / \Lambda_{R}\right)$ is the Lamé constant at $t=\infty$.

It is worth noticing that, differently from the plate model, now viscosity changes the stiffness in a nonproportional way, since the body is considered viscously volumetrically incompressible; for the same reason, damping is of nonproportional type. 


\section{Conclusions}

A linear metamodel of viscoelastic continuum has been formulated. It is expressed in terms of internal variables, having the meaning of the viscous elongations of the (arbitrary in number) dashpots of the underlying rheological model. It was observed that, due to the smallness of damping, the eigenvalue spectrum is well separated, so it entails that the dynamics is rapidly attracted by the space spanned by the (finite-dimensional) central eigenvectors. Two alternative algorithms, based on the center manifold method and the multiple scales method, have been worked out to derive the reduced system. Both methods avoid the evaluation of the central eigenvectors, as instead needed in bifurcation theory. The reduction allows eliminating the (passive) internal variables, while accounting for them as slaves of the (active) observable variables.

Three sample continua of increasing spatial dimensions have been considered for illustrative purposes, namely (a) the transverse vibrations of an Euler-Bernoulli beam resting on a Winkler-type soil, the viscoelastic properties of both being described by the standard three-parameter viscoelastic model, (b) the transverse vibrations of a Kirchhoff plate, obeying to the five-parameter viscoelastic solid model, and (c) a three-dimensional Cauchy continuum, whose volumetric strains are elastic and deviatoric strains viscoelastic. The following conclusions can be drawn.

(1) The lowest-order approximation of the center subspaces supplies a reduced stiffness operator which coincides with the elastic one, but with elastic moduli replaced by the equilibrium values (at infinite time) of the relaxation moduli. This is due to the fact that low damping entails short relaxation times, on the order of the transient duration, so that the steady dynamics occurs when the moduli reach their asymptotic values.

(2) The reduced damping operator is made of a linear combination, with relaxation moduli, of the derivatives which form the stiffness operator. In general, however, damping and stiffness are not proportional, so the Rayleigh simplistic model of damping is not recovered.

(3) The beam on viscoelastic soil can be reduced to an internally and externally damped beam on elastic Winkler soil. Reduction provides the equivalent characteristics. Since viscosity in the two substructures acts independently, the resulting damping is nonproportional. However, if the beam is in air (no soil), damping is of proportional type. A formula has been given for practical design of damping.

(4) The viscoelastic five-parameter plate shows the power of the model, which permits elimination of six internal variables (two for each of the three curvatures), and a strong lowering of dimensions. Due to the fact that the same 
constitutive law has been adopted for all the components of stress, a damping operator proportional to the stiffness has been found.

(5) The three-dimensional Cauchy continuum, in which viscous incompressibility has been adopted, can also be reduced to an internally damped continuum, expressed in terms of displacements and velocities only. The elastic part of the equation consists of the classical Navier equation, in which, however, the Lamé constants are modified as their value at infinite time, in a nonproportional way, due to the different behavior of the deviatoric and spherical parts. Damping is found to be of nonproportional type.

\section{Appendix: A microstructured homogenized one-dimensional continuum}

The chain of oscillators displayed in Figure 4, top, made of $n$ cells of length $h$, is considered. Each cell is a standard three-parameter viscoelastic oscillator, carrying a mass $M$, whose constitutive parameters $c_{0}, c_{v}$, and $b$ are constant along the chain. A homogenization procedure is here developed to obtain an equivalent continuum, representative of the behavior of the chain.

The free dynamics of the continuum is then analyzed under the assumption of small viscous modulus, by making use of asymptotic solutions. It will be shown that a suitable design of the microstructure could lead to spectral properties of the continuum somewhat analogous to those discussed in Section 3.

The considered target continuum is a viscoelastic one-dimensional model, i.e., a bar of length $\ell=n h$ and mass per unit length $m=M / h$ (Figure 4, bottom). In what follows the chain is referred to as the fine model and the homogeneous continuum as the coarse model.

The constitutive law of the periodic structure. The $i$-th cell (Figure 5, left) is taken as the representative volume of the periodic structure. Its constitutive law can

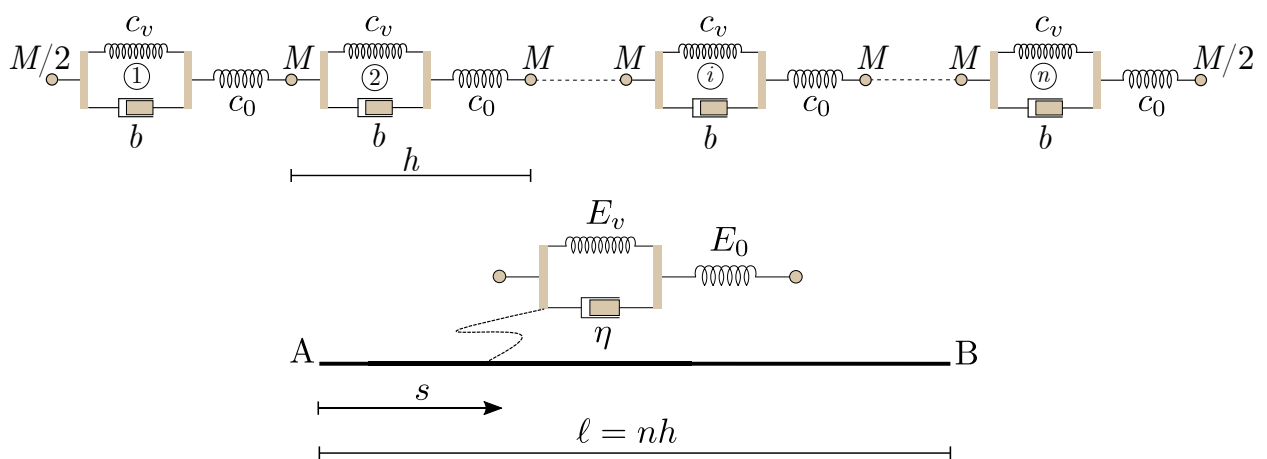

Figure 4. Viscoelastic one-dimensional systems: fine discrete periodic model (top) and homogeneous bar (bottom). 

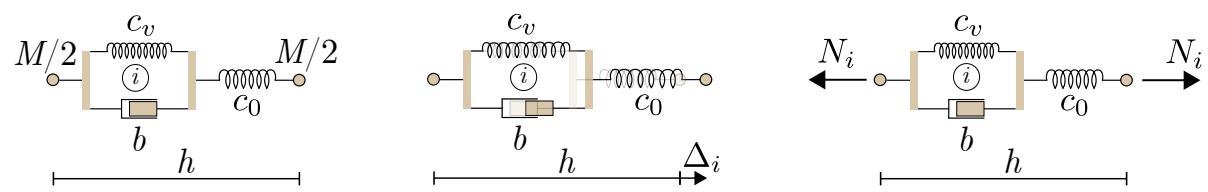

Figure 5. Fine model: cell (left), kinematics (center), and statics (right).

be written by rearranging kinematics (Figure 5, center) and equilibrium (Figure 5, right) equations of the rheological model [Luongo and D'Annibale 2017a]. It reads

$$
\begin{aligned}
N_{i} & =c_{0}\left(\Delta_{i}-\kappa_{i}\right), \\
\dot{\kappa}_{i} & =\frac{c_{0}}{b} \Delta_{i}-\frac{c_{0}+c_{v}}{b} \kappa_{i}
\end{aligned}
$$

where $N_{i}$ is the axial force and $\Delta_{i}$ and $\kappa_{i}$ are the total and viscous displacements, respectively.

The viscoelastic one-dimensional continuum model. The axial motion of a viscoelastic one-dimensional bar is then considered (Figure 4, bottom). Kinematics and equilibrium are described by

$$
\begin{aligned}
u^{\prime} & =\varepsilon, \\
-N^{\prime} & =f-m \ddot{u}
\end{aligned}
$$

where $u(s, t), \varepsilon(s, t), N(s, t)$, and $f(s, t)$ are the axial displacement, the unit extension, the cross-section normal force, and the horizontal external load, respectively, which are functions of the abscissa $s$ and time $t$. Moreover, the prime and the dot denote spatial and time differentiation, respectively. It is worth noticing that (69) are in the same form as (1).

According to the standard three-parameter model, the constitutive law reads

$$
\begin{aligned}
N & =E_{0} A\left(\varepsilon-\varepsilon_{v}\right), \\
\dot{\varepsilon}_{v} & =\frac{E_{0}}{\eta} \varepsilon-\frac{E_{0}+E_{v}}{\eta} \varepsilon_{v}
\end{aligned}
$$

where $E_{0}, E_{v}, \eta$ are material constants, $\varepsilon_{v}$ is an internal variable having the meaning of viscous extensional strain, and $A$ is the area of the cross-section. It is important to remark that (70) are in the same form as (2) of the metamodel.

The constitutive coefficients of the coarse model are determined in the framework of the homogenization procedure. In particular, this is carried out by exploiting the periodicity of the fine model and following the next steps: (i) the constitutive law for one of its cells (68) is written in terms of strains of the coarse model, thus entailing $\Delta_{i}=\varepsilon h$ and $\kappa_{i}=\varepsilon_{v} h$ and (ii) the constitutive law of the cell is attributed 
to a slice of the equivalent bar of the same length. The following identification of the coefficients of the equivalent bar holds:

$$
E_{0} A:=h c_{0}, \quad \frac{E_{0}}{\eta}:=\frac{c_{0}}{b}, \quad \frac{E_{v}}{\eta}:=\frac{c_{v}}{b} .
$$

By combining (69) and (70) in the state space form $(\dot{u}=v)$, the equations of motion of the bar read

$$
\left(\begin{array}{c}
\dot{u} \\
\dot{v} \\
\dot{\varepsilon}_{v}
\end{array}\right)=\left[\begin{array}{ccc}
0 & 1 & 0 \\
\left(E_{0} A / m\right) \partial_{s}^{2} & 0 & -\left(E_{0} A / m\right) \partial_{s}^{1} \\
\left(E_{0} / \eta\right) \partial_{s}^{1} & 0 & -\left(E_{0}+E_{v}\right) / \eta
\end{array}\right]\left(\begin{array}{c}
u \\
v \\
\varepsilon_{v}
\end{array}\right)+\left(\begin{array}{c}
0 \\
f / m \\
0
\end{array}\right) .
$$

These equation are in the form of (3). They must be integrated with initial conditions (assuming the system is initially at rest). Moreover, it is assumed that geometric boundary conditions at the ends prescribe null horizontal displacement, i.e., $u_{\mathrm{A}}=u_{\mathrm{B}}=0$.

Free dynamics. The solution of (72), which (exactly) satisfies the spatial eigenvalue problem, is in the form

$$
\left(\begin{array}{l}
u \\
v
\end{array}\right)=\left(\begin{array}{c}
\hat{u}(t) \\
\hat{v}(t)
\end{array}\right) \sin \left(\frac{k \pi s}{\ell}\right), \quad \varepsilon_{v}=\hat{\varepsilon}_{v}(t) \cos \left(\frac{k \pi s}{\ell}\right)
$$

where $\hat{u}(t), \hat{v}(t)$, and $\hat{\varepsilon}_{v}(t)$ are (time-dependent) amplitudes and $k$ denotes the wave number. Therefore, the associated characteristic equation of the (unforced) system (72) reads

$$
\eta \lambda^{3}+\left(E_{0}+E_{v}\right) \lambda^{2}+\frac{\eta E_{0} A}{m}\left(\frac{k \pi}{\ell}\right)^{2} \lambda+\frac{E_{0} E_{v} A}{m}\left(\frac{k \pi}{\ell}\right)^{2}=0 .
$$

Approximated roots of (74) in the case $\eta$ is small can be determined through a perturbation method (details not reported here). It is found that

$$
\begin{aligned}
\lambda_{1}=-\frac{E_{0}+E_{v}}{\eta}+O(1) & =:-\frac{1}{t_{\mathrm{rel}}}, \\
\lambda_{2,3} & =-\frac{\eta E_{0}^{2} A}{2 m\left(E_{0}+E_{v}\right)^{2}}\left(\frac{k \pi}{\ell}\right)^{2} \pm i \sqrt{\frac{E_{0} E_{v} A}{m\left(E_{0}+E_{v}\right)}\left(\frac{k \pi}{\ell}\right)^{2}}+\mathcal{O}\left(\epsilon^{2}\right)=:-\xi \omega \pm i \omega
\end{aligned}
$$

where $t_{\text {rel }}$ is the relaxation time of the standard three-parameter model. In particular, accordingly to the discussion of Section 3 , it can be seen that one eigenvalue $\lambda_{1}$ is real, of order $\epsilon^{-1}$, and its magnitude is inversely proportional to $t_{\text {rel }}$, while the other two eigenvalues $\lambda_{2}$ and $\lambda_{3}$ are complex-conjugate with order- $\epsilon$ negative real part. Moreover, the following positions hold:

$$
\omega^{2}:=\frac{E_{\infty} A}{m}\left(\frac{k \pi}{\ell}\right)^{2}, \quad \xi:=\frac{\eta E_{0}^{2} A}{2 m \omega\left(E_{0}+E_{v}\right)^{2}}\left(\frac{k \pi}{\ell}\right)^{2},
$$




\begin{tabular}{|ccccc|}
\hline$M[\mathrm{~kg}]$ & $h[\mathrm{~m}]$ & $c_{0}[\mathrm{~N} / \mathrm{m}]$ & $c_{v}[\mathrm{~N} / \mathrm{m}]$ & $b[\mathrm{Ns} / \mathrm{m}]$ \\
1 & 0.2 & 800 & 800 & 50 \\
\hline
\end{tabular}

Table 1. Numerical values of the parameters of the microstructure.

$E_{\infty}:=E_{0} E_{v} /\left(E_{0}+E_{v}\right)$ being the elastic modulus at infinite time. As shown in [Luongo and D'Annibale 2017a], definitions (76) represent the (undamped) frequency and damping ratio of a reduced oscillator, which is able to recover the oscillatory dynamics of the bar after the exponential transient motion decays, and whose motion is governed by the equation

$$
\ddot{u}+2 \xi \omega \dot{u}+\omega^{2} u=\frac{f}{m} .
$$

Discussion about the validity of the perturbation solution. The ratio between the moduli of the real parts of eigenvalues (75) can be written as

$$
r:=\frac{\left|\operatorname{Re} \lambda_{2,3}\right|}{\left|\operatorname{Re} \lambda_{1}\right|}=\frac{E_{0} \omega^{2}}{2 E_{v}} t_{\mathrm{rel}}^{2} .
$$

In particular, by considering $E_{v}$ and $E_{0}$ of the same order of magnitude, e.g., $E_{v}=$ $E_{0} / 2$,

$$
r=\omega^{2} t_{\mathrm{rel}}^{2} \simeq 40\left(\frac{t_{\mathrm{rel}}}{T}\right)^{2}, \quad t_{\mathrm{rel}}=\frac{2}{3} \frac{\eta}{E_{0}},
$$

$T:=2 \pi / \omega$ being the (undamped) period of the structure. By requiring the spectrum of eigenvalues to be well separated, i.e., $r=\mathscr{O}\left(\epsilon^{2}\right)=10^{-2}$, it is found that

$$
\frac{t_{\text {rel }}}{T} \simeq \frac{1}{65} \text {. }
$$

It means that, if the period of the structure is on the order of the second, as occurs in civil structures, $\eta$ must be small, i.e., $\eta \simeq 0.02 E_{0}$. However, the relaxation time of a structural material, as, e.g., identified by a relaxation test, is found of some order of magnitude greater with respect to the structural period. For example for rubber $t_{\text {rel }} \sim 100 \mathrm{~s}$, thus entailing $\eta$ large $\left(\eta \simeq 150 E_{0}\right)$. It is concluded that a well separated spectrum is representative of those viscoelastic structures, in which the relaxation time of the underlying viscoelastic constitutive model is sufficiently smaller than their fundamental period.

The ratio (80) can be easily achieved in the microstructured continuum, by designing its microstructure. As an example, by taking the numerical values reported in Table 1, and $n=20$, the coefficients of Table 2 follow for the equivalent bar. It is found that the first (undamped) period of the structure is $T=2 \mathrm{~s}$, and its relaxation time $t_{\text {rel }}=\frac{1}{32} \mathrm{~s}$, thus corresponding to $t_{\text {rel }} / T=\frac{1}{64}$. Finally, in Figure 6 the very good agreement between the complex-conjugate exact eigenvalues (gray circles) 


\begin{tabular}{|ccccc|}
\hline$m[\mathrm{~kg} / \mathrm{m}]$ & $\ell[\mathrm{m}]$ & $E_{0} A[\mathrm{~N}]$ & $E_{0} / \eta\left[\mathrm{s}^{-1}\right]$ & $E_{v} / \eta\left[\mathrm{s}^{-1}\right]$ \\
5 & 4 & 160 & 16 & 16 \\
\hline
\end{tabular}

Table 2. Numerical values of the parameters of the equivalent bar.

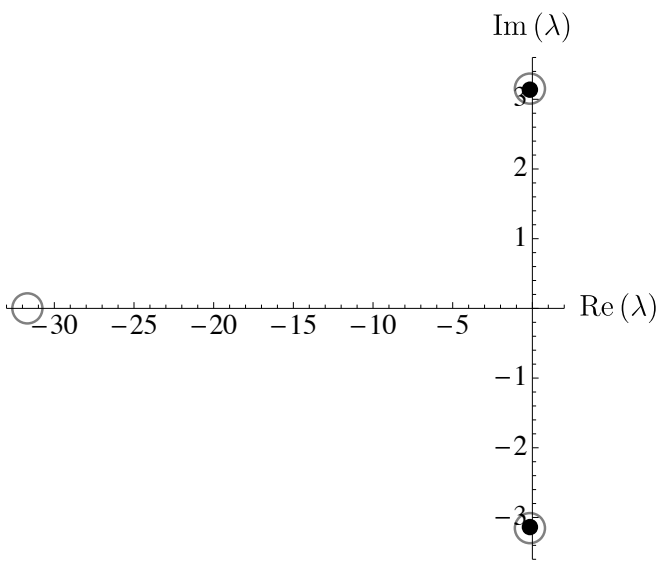

Figure 6. Eigenvalues of the exact (gray circles) and reduced (black dots) systems.

and the asymptotic ones (black dots), obtained by the (unforced) (77), is shown when $k=1$. The exact real eigenvalue is filtered by the reduction procedure.

\section{References}

[Altenbach and Eremeyev 2015] H. Altenbach and V. A. Eremeyev, "On the constitutive equations of viscoelastic micropolar plates and shells of differential type", Math. Mech. Complex Syst. 3:3 (2015), 273-283.

[Barchiesi et al. 2019] E. Barchiesi, M. Spagnuolo, and L. Placidi, "Mechanical metamaterials: a state of the art", Math. Mech. Solids 24:1 (2019), 212-234.

[Baroudi et al. 2019] D. Baroudi, I. Giorgio, A. Battista, E. Turco, and L. A. Igumnov, "Nonlinear dynamics of uniformly loaded Elastica: Experimental and numerical evidence of motion around curled stable equilibrium configurations", Z. Angew. Math. Mech. 99:7 (2019), art. id. e201800121.

[Del Vescovo and Giorgio 2014] D. Del Vescovo and I. Giorgio, "Dynamic problems for metamaterials: review of existing models and ideas for further research", Internat. J. Engrg. Sci. 80 (2014), 153-172.

[Eugster et al. 2019] S. R. Eugster, F. dell'Isola, and D. J. Steigmann, "Continuum theory for mechanical metamaterials with a cubic lattice substructure”, Math. Mech. Complex Syst. 7:1 (2019), 75-98.

[Flügge 1975] W. Flügge, Viscoelasticity, Springer, 1975.

[Frazier and Hussein 2015] M. J. Frazier and M. I. Hussein, "Viscous-to-viscoelastic transition in phononic crystal and metamaterial band structures", J. Acoust. Soc. Am. 138:5 (2015), 3169-3180.

[Fusco and Hale 1989] G. Fusco and J. K. Hale, "Slow-motion manifolds, dormant instability, and singular perturbations", J. Dynam. Differential Equations 1:1 (1989), 75-94. 
[Giorgio et al. 2017] I. Giorgio, U. Andreaus, F. dell'Isola, and T. Lekszycki, "Viscous second gradient porous materials for bones reconstructed with bio-resorbable grafts", Extreme Mech. Lett. 13 (2017), 141-147.

[Golla and Hughes 1985] D. F. Golla and P. C. Hughes, "Dynamics of viscoelastic structures - a time-domain, finite element formulation", J. Appl. Mech. 52:4 (1985), 897-906.

[Guckenheimer and Holmes 1983] J. Guckenheimer and P. Holmes, Nonlinear oscillations, dynamical systems, and bifurcations of vector fields, Applied Mathematical Sciences 42, Springer, 1983.

[Hatada et al. 2000] T. Hatada, T. Kobori, M. Ishida, and N. Niwa, "Dynamic analysis of structures with Maxwell model”, Earthq. Eng. Struct. D. 29:2 (2000), 159-176.

[Lekszycki et al. 1992] T. Lekszycki, N. Olhoff, and J. J. Pedersen, "Modelling and identification of viscoelastic properties of vibrating sandwich beams", Compos. Struct. 22:1 (1992), 15-31.

[Lemaitre and Chaboche 1990] J. Lemaitre and J.-L. Chaboche, Mechanics of solid materials, Cambridge University, 1990.

[Lewandowski et al. 2012] R. Lewandowski, A. Bartkowiak, and H. Maciejewski, "Dynamic analysis of frames with viscoelastic dampers: a comparison of damper models", Struct. Eng. Mech. 41:1 (2012), 113-137.

[Lewińska et al. 2017] M. A. Lewińska, V. G. Kouznetsova, J. A. W. van Dommelen, A. O. Krushynska, and M. G. D. Geers, "The attenuation performance of locally resonant acoustic metamaterials based on generalised viscoelastic modelling”, Int. J. Solids Struct. 126-127 (2017), 163-174.

[Luongo and D'Annibale 2013] A. Luongo and F. D'Annibale, "Double zero bifurcation of nonlinear viscoelastic beams under conservative and non-conservative loads", Int. J. Non-Lin. Mech. 55 (2013), 128-139.

[Luongo and D'Annibale 2017a] A. Luongo and F. D'Annibale, “Invariant subspace reduction for linear dynamic analysis of finite-dimensional viscoelastic structures", Meccanica 52:13 (2017), 3061-3085.

[Luongo and D'Annibale 2017b] A. Luongo and F. D'Annibale, "Nonlinear hysteretic damping effects on the post-critical behaviour of the visco-elastic Beck's beam", Math. Mech. Solids 22:6 (2017), 1347-1365.

[Luongo and Paolone 1999] A. Luongo and A. Paolone, "On the reconstitution problem in the multiple time-scale method", Nonlinear Dynam. 19:2 (1999), 133-156.

[Luongo and Zulli 2012a] A. Luongo and D. Zulli, "Dynamic analysis of externally excited NEScontrolled systems via a mixed multiple scale/harmonic balance algorithm", Nonlinear Dynam. 70:3 (2012), 2049-2061.

[Luongo and Zulli 2012b] A. Luongo and D. Zulli, "A paradigmatic system to study the transition from zero/Hopf to double-zero/Hopf bifurcation”, Nonlinear Dynam. 70:1 (2012), 111-124.

[Luongo and Zulli 2014] A. Luongo and D. Zulli, "Aeroelastic instability analysis of NES-controlled systems via a mixed multiple scale/harmonic balance method", J. Vib. Control 20:13 (2014), 19851988.

[Luongo et al. 2016] A. Luongo, F. D'Annibale, and M. Ferretti, "Hard loss of stability of Ziegler's column with nonlinear damping", Meccanica 51:11 (2016), 2647-2663.

[Manimala and Sun 2014] J. M. Manimala and C. T. Sun, "Microstructural design studies for locally dissipative acoustic metamaterials", J. Appl. Phys. 115:2 (2014), art. id. 023518.

[Maugin and Muschik 1990] G. A. Maugin and W. Muschik, "Thermodynamics with internal variables, I: General concepts”, J. Non-Equil. Thermod. 19:3 (1990), 217-249. 
[McTavish and Hughes 1993] D. J. McTavish and P. C. Hughes, "Modeling of linear viscoelastic space structures", J. Vib. Acoust. 115:1 (1993), 103-110.

[Moreau 1970] J. J. Moreau, "Sur les lois de frottement, de plasticité et de viscosité", C. R. Acad. Sci. Paris A 271 (1970), 608-611.

[Muscolino and Palmeri 2007] G. Muscolino and A. Palmeri, "Response of beams resting on viscoelastically damped foundation to moving oscillators", Int. J. Solids Struct. 44:5 (2007), 13171336.

[Narayanan and Beskos 1982] G. V. Narayanan and D. E. Beskos, "Numerical operational methods for time-dependent linear problems", Internat. J. Numer. Methods Engrg. 18:12 (1982), 1829-1854.

[Nayfeh 2000] A. H. Nayfeh, Perturbation methods, Wiley, New York, 2000.

[Nayfeh and Mook 1979] A. H. Nayfeh and D. T. Mook, Nonlinear oscillations, Wiley, New York, 1979.

[Schanz 1999] M. Schanz, "A boundary element formulation in time domain for viscoelastic solids", Comm. Numer. Methods Engrg. 15:11 (1999), 799-809.

[Schanz 2001] M. Schanz, Wave propagation in viscoelastic and poroelastic continua: a boundary element approach, Lecture Notes in Applied Mechanics 2, Springer, 2001.

[Shaw and Pierre 1991] S. W. Shaw and C. Pierre, "Nonlinear normal modes and invariant manifolds", J. Sound Vib. 150:1 (1991), 170-173.

[Sim and Kwak 1988] W. J. Sim and B. M. Kwak, "Linear viscoelastic analysis in time domain by boundary element method”, Comput. Struct. 29:4 (1988), 531-539.

[Syngellakis 2003] S. Syngellakis, "Boundary element methods for polymer analysis", Eng. Anal. Bound. Elem. 27:2 (2003), 125-135.

[Troger and Steindl 1991] H. Troger and A. Steindl, Nonlinear stability and bifurcation theory: an introduction for engineers and applied scientists, Springer, 1991.

[Wiggins 2003] S. Wiggins, Introduction to applied nonlinear dynamical systems and chaos, 2nd ed., Texts in Applied Mathematics 2, Springer, 2003.

Received 21 Oct 2019. Revised 9 Jan 2020. Accepted 18 Feb 2020.

ANGELO LUONGO: angelo.luongo@univaq.it

Dipartimento di Ingegneria Civile, Edile-Architettura e Ambientale, Il Centro Internazionale di Ricerca per la Matematica e Meccanica dei Sistemi Complessi, Università degli Studi dell'Aquila, L'Aquila, Italy

FRANCESCO D'ANNIBALE: francesco.dannibale@univaq.it

Dipartimento di Ingegneria Civile, Edile-Architettura e Ambientale, Il Centro Internazionale di Ricerca per la Matematica e Meccanica dei Sistemi Complessi, Università degli Studi dell'Aquila, L'Aquila, Italy 
EDITORIAL BOARD

ANTONIO CARCATERRA

ERIC A. CARLEN

FRANCESCO DELL'ISOLA

RAFFAELE ESPOSITO

ALBERT FANNJIANG

Gilles A. FranCFORT

PiERANGELO MARCATI

JEAN-JACQUES MARIGO

PETER A. MARKOWICH

MARTIN OSTOJA-STARZEWSKI

PIERRE SEPPECHER

DAVID J. STEIGMANN

PAUl STEINMANN

PierRe M. SuQueT

MANAGING EDITORS

MICOL AMAR

EMILIO BARCHIESI

ANGELA MADEO

MARTIN OSTOJA-STARZEWSKI

ADVISORY BOARD

ADNAN AKAY

Holm AltenbaCH

MICOL AMAR

HARM ASKES

TEODOR ATANACKOVIĆ

VICTOR BERDICHEVSKY

GUY BOUCHITTÉ

ANDREA BRAIDES

ROBERTO CAMASSA

MAURO CARFORE

ERIC DARVE

FELIX DARVE

ANNA DE MASI

Gianpietro Del Piero

EMMANUELE Di BENEDETTO

VICTOR A. EREMEYEV

BERNOLD FIEDLER

IRENE M. GAMBA

DAVID Y. GAO

Sergey GaVRILyuk

Timothy J. HEALEY

DOMINIQUe JEULiN

ROGER E. KHAYAT

Corrado LATTANZIO

ROBERT P. LIPTON

ANGELO LUONGO

ANGELA MADEO

JUAN J. MANFREDI

CARLO MARCHIORO ANIL MisRa

ROBERTO NATALINI PATRIZIO NEFF

Thomas J. PENCE

ANDREY PIATNITSKI

ERRICO PRESUTTI

MARIO Pulvirenti

LUCIO RUSSO

Miguel A. F. SAnjuaN

PATRICK SElVAdurai

MiroslaV ŠILHAVÝ

GUIDO SWEERS

ANTOINETTE TORDESILLAS

LEV TRUSKINOVSKY

JUAN J. L. VELÁZQUEZ

VINCENZO VESPRI

ANGELO VULPIANI msp.org/memocs

Università di Roma "La Sapienza", Italia

Rutgers University, USA

(CO-CHAIR) Università di Roma "La Sapienza", Italia

(TREASURER) Università dell'Aquila, Italia

University of California at Davis, USA

(CO-CHAIR) Université Paris-Nord, France

Università dell' Aquila, Italy

École Polytechnique, France

DAMTP Cambridge, UK, and University of Vienna, Austria

(CHAIR MANAGING EDITOR) Univ. of Illinois at Urbana-Champaign, USA

Université du Sud Toulon-Var, France

University of California at Berkeley, USA

Universität Erlangen-Nürnberg, Germany

LMA CNRS Marseille, France

Università di Roma "La Sapienza”, Italia

Università degli Studi dell'Aquila, Italy

Université de Lyon-INSA (Institut National des Sciences Appliquées), France

(CHAIR MANAGING EDITOR) Univ. of Illinois at Urbana-Champaign, USA

Carnegie Mellon University, USA, and Bilkent University, Turkey

Otto-von-Guericke-Universität Magdeburg, Germany

Università di Roma "La Sapienza", Italia

University of Sheffield, UK

University of Novi Sad, Serbia

Wayne State University, USA

Université du Sud Toulon-Var, France

Università di Roma Tor Vergata, Italia

University of North Carolina at Chapel Hill, USA

Università di Pavia, Italia

Stanford University, USA

Institut Polytechnique de Grenoble, France

Università dell'Aquila, Italia

Università di Ferrara and International Research Center MEMOCS, Italia

Vanderbilt University, USA

Gdansk University of Technology, Poland

Freie Universität Berlin, Germany

University of Texas at Austin, USA

Federation University and Australian National University, Australia

Université Aix-Marseille, France

Cornell University, USA

École des Mines, France

University of Western Ontario, Canada

Università dell' Aquila, Italy

Louisiana State University, USA

Università dell'Aquila, Italia

Université de Lyon-INSA (Institut National des Sciences Appliquées), France

University of Pittsburgh, USA

Università di Roma "La Sapienza", Italia

University of Kansas, USA

Istituto per le Applicazioni del Calcolo "M. Picone", Italy

Universität Duisburg-Essen, Germany

Michigan State University, USA

Narvik University College, Norway, Russia

Università di Roma Tor Vergata, Italy

Università di Roma "La Sapienza", Italia

Università di Roma "Tor Vergata", Italia

Universidad Rey Juan Carlos, Madrid, Spain

McGill University, Canada

Academy of Sciences of the Czech Republic

Universität zu Köln, Germany

University of Melbourne, Australia

École Polytechnique, France

Bonn University, Germany

Università di Firenze, Italia

Università di Roma La Sapienza, Italia

MEMOCS (ISSN 2325-3444 electronic, 2326-7186 printed) is a journal of the International Research Center for the Mathematics and Mechanics of Complex Systems at the Università dell'Aquila, Italy.

Cover image: "Tangle” by @ John Horigan; produced using the Context Free program (contextfreeart.org).

\section{PUBLISHED BY}

\section{I. mathematical sciences publishers} nonprofit scientific publishing http://msp.org/ 
Mathematics and Mechanics of Complex Systems vol. 8 no. 2

Genotype-dependent virus distribution and competition of 101 virus strains

Nikolai Bessonov, Gennady A. Bocharov, Cristina Leon, Vladimir Popov and Vitaly Volpert

Modeling the linear dynamics of continuous viscoelastic systems on their infinite-dimensional central subspace

Angelo Luongo and Francesco D'Annibale

The method of virtual power in the mechanics of continuous media, I: Second-gradient theory

\section{Paul Germain}

An appreciation and discussion of Paul Germain's "The method of virtual power in the mechanics of continuous media, I: Second-gradient theory"

Marcelo Epstein and Ronald E. Smelser

MEMOCS is a journal of the International Research Center for the Mathematics and Mechanics of Complex Systems at the Università dell' Aquila, Italy.

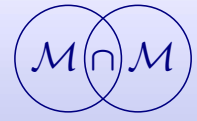

